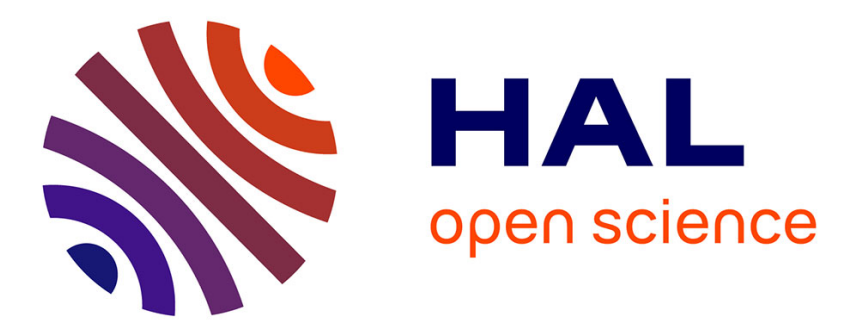

\title{
Macroscopic behavior and field fluctuations in viscoplastic composites: Second-order estimates versus full-field simulations
}

\author{
Martin Idiart, Hervé Moulinec, Pedro Ponte Castañeda, Pierre Suquet
}

\section{- To cite this version:}

Martin Idiart, Hervé Moulinec, Pedro Ponte Castañeda, Pierre Suquet. Macroscopic behavior and field fluctuations in viscoplastic composites: Second-order estimates versus full-field simulations. Journal of the Mechanics and Physics of Solids, 2006, 6, pp.201-208. 10.1016/j.jmps.2005.11.004 hal-00111463

\author{
HAL Id: hal-00111463 \\ https://hal.science/hal-00111463
}

Submitted on 8 Jul 2019

HAL is a multi-disciplinary open access archive for the deposit and dissemination of scientific research documents, whether they are published or not. The documents may come from teaching and research institutions in France or abroad, or from public or private research centers.
L'archive ouverte pluridisciplinaire HAL, est destinée au dépôt et à la diffusion de documents scientifiques de niveau recherche, publiés ou non, émanant des établissements d'enseignement et de recherche français ou étrangers, des laboratoires publics ou privés. 


\title{
Macroscopic behavior and field fluctuations in viscoplastic composites: Second-order estimates versus full-field simulations
}

\author{
M.I. Idiart ${ }^{\mathrm{a}, \mathrm{b}}, \mathrm{H}$. Moulinec ${ }^{\mathrm{c}}$, P. Ponte Castañeda ${ }^{\mathrm{a}, \mathrm{b}, *}$, P. Suquet ${ }^{\mathrm{c}}$ \\ ${ }^{a}$ Laboratoire de Mécanique des Solides, C.N.R.S. UMR 7649, Département de Mécanique, École Polytechnique, \\ 91128 Palaiseau Cedex, France \\ ${ }^{\mathrm{b}}$ Department of Mechanical Engineering and Applied Mechanics, University of Pennsylvania, Philadelphia, \\ PA 19104-6315, USA \\ ${ }^{\mathrm{c}}$ L.M.A./C.N.R.S. UPR 7051, 31 Chemin Joseph Aiguier, 13402 Marseille Cedex 20, France
}

\begin{abstract}
This work presents a combined numerical and theoretical study of the effective behavior and statistics of the local fields in random viscoplastic composites. The full-field numerical simulations are based on the fast Fourier transform (FFT) algorithm [Moulinec, H., Suquet, P., 1994. A fast numerical method for computing the linear and nonlinear properties of composites. C. R. Acad. Sci. Paris II 318, 1417-1423], while the theoretical estimates follow from the so-called "second-order" procedure [Ponte Castañeda, P., 2002a. Second-order homogenization estimates for nonlinear composites incorporating field fluctuations: I-Theory. J. Mech. Phys. Solids 50, 737-757]. Twophase fiber composites with power-law phases are considered in detail, for two different heterogeneity contrasts corresponding to fiber-reinforced and fiber-weakened composites. Both the FFT simulations and the corresponding "second-order" estimates show that the strain-rate fluctuations in these systems increase significantly, becoming progressively more anisotropic, with increasing nonlinearity. In fact, the strain-rate fluctuations tend to become unbounded in the limiting case of ideally plastic composites. This phenomenon is shown to correspond to the localization of the strain field into bands running through the composite along certain preferred orientations determined by the loading conditions. The bands tend to avoid the fibers when they are stronger than the matrix, and to pass through the fibers when they are weaker than the matrix. In general, the
\end{abstract}

\footnotetext{
${ }^{*}$ Corresponding author. Department of Mechanical Engineering and Applied Mechanics, University of Pennsylvania, 220 S. 33rd Street, Philadelphia, PA 19104-6315, USA. Tel.: + 12158985046 ; fax: + 12155736334.

E-mail address: ponte@seas.upenn.edu (P. Ponte Castañeda).
} 
"second-order" estimates are found to be in good agreement with the FFT simulations, even for high nonlinearities, and they improve, often in qualitative terms, on earlier nonlinear homogenization estimates. Thus, it is demonstrated that the "second-order" method can be used to extract accurate information not only for the macroscopic behavior, but also for the anisotropic distribution of the local fields in nonlinear composites.

Keywords: Creep; Inhomogeneous material; Viscoplastic material; Homogenization; Numerical algorithms

\section{Introduction}

Homogenization methods for nonlinear heterogeneous media have come a long way since the early work of Taylor (1938) to estimate the effective behavior of viscoplastic polycrystals. Following the development of rigorous bounds by Talbot and Willis (1985), who made use of an extension of the Hashin-Shtrikman variational principles for nonlinear media (Willis, 1983), a novel "variational" method was proposed by Ponte Castañeda (1991) to generate more general types of estimates for the effective behavior of nonlinear composites in terms of suitably optimized linear comparison composites (LCCs). Closely related methods have been developed by Suquet (1993) for the special cases of power-law composites. Suquet (1995) and $\mathrm{Hu}$ (1996) have shown that the "variational" method of Ponte Castañeda (1991) could be given an alternative interpretation as a "modified secant" approach, thus establishing a link with the second moments of the local fields in the LCC. In an attempt to improve on these "variational" estimates, and in particular, to generate estimates that are exact to second order in the heterogeneity contrast, and therefore agree with the perturbation expansions of Suquet and Ponte Castañeda (1993) for weakly inhomogeneous nonlinear composites, Ponte Castañeda (1996) proposed a "second-order" method, which made use of an LCC with elastic moduli given by the tangent moduli of the nonlinear phases evaluated at the phase averages of the fields in the LCC. However, this method may violate the rigorous bounds provided by the earlier "variational" method when the field fluctuations are large, as is the case near the percolation limit. Motivated by this finding, Ponte Castañeda (2002a) proposed an improved "second-order" method that makes use of a "generalized secant" interpolation of the nonlinear constitutive relations, incorporating dependence on both the first and second moments of the relevant fields in the LCCs.

In parallel developments, methods have also been developed for computing numerically the effective behavior of nonlinear composites. One of these methods that is particularly well suited to strongly nonlinear composites, such as viscoplastic materials with low strainrate sensitivity, or in the limit, ideally plastic materials, is the fast Fourier transform (FFT) method first proposed by Moulinec and Suquet (1994, 1998), and developed further by Michel et al. (2001). Such accurate numerical simulations allow for, among other things, comparisons with the above-mentioned theoretical approaches with the objective of assessing the accuracy of the latter. Comparisons of this type have already been carried out (Moulinec and Suquet, 2003, 2004) in the context of the earlier "variational" method showing that, while more accurate in general than earlier methods, the "variational" method can lead to inaccurate predictions for large values of the heterogeneity parameter and strong nonlinearities. One of the goals of this study is to investigate the accuracy of the "second-order" estimates, particularly with reference to the earlier variational approach. 
In the development of the "second-order" method, it was recognized (Ponte Castañeda, 2002a) that this method could not only be used to generate estimates for the effective behavior of the nonlinear composite, but also to extract estimates for the covariance of the field fluctuations in the phases of the nonlinear composite. Such information could be useful for obtaining improved descriptions of microstructure evolution in composite materials and polycrystals that are subjected to finite-deformation processes, as well as for developing statistical theories of damage nucleation and evolution in heterogeneous material systems. Field fluctuations in linear composites have been studied by Bobeth and Diener (1987), Parton and Buryachenko (1990), and Cheng and Torquato (1997), among others. A combined experimental and theoretical study of field fluctuations in two-phase elastoplastic solids was given by Bornert et al. (1994). In the context of conductivity, Pellegrini (2000) proposed that a Gaussian distribution of the local fields in the constituent phases of random composites with linear constitutive behavior could be a good approximation, and used this type of distribution as an ansatz for random composites with nonlinear constitutive behavior, in an attempt to account for field fluctuations in selfconsistent nonlinear homogenization methods (Pellegrini, 2001). Another objective of this work is to investigate this point further.

In connection with applications of the "second-order" method to porous and two-phase power-law composites, Ponte Castañeda (2002b) and Idiart and Ponte Castañeda (2003) found that the covariance of the field fluctuations generally increase and become highly anisotropic with increasing nonlinearity. The conjecture was then made in these works that the developing anisotropy of the field fluctuations with nonlinearity could be correlated with the known fact that the strain fields become localized and preferentially oriented for strongly nonlinear composites (e.g., Moulinec and Suquet, 1998). Of course, the numerical simulations allow for the characterization of the full fields in the composite, and it is natural to compute, in particular, the covariance of the field fluctuations in the phases of the nonlinear composite, and to compare them with the theoretical predictions, in an attempt to assess whether the homogenization theories can be used to reliably extract information about the field fluctuations. A preliminary study of such comparisons has been carried out recently by Moulinec and Suquet $(2003,2004)$ in the context of the isotropic "variational" method, and it has been shown there that the predictions of this method for the second moments of the fields are not accurate.

In the present work, comparisons will be made between the theoretical predictions of the "second-order" method for the averages and standard deviations of the fields in the phases of a certain class of nonlinear composites with particulate microstructure, and the corresponding results from the full-field numerical simulations. The objective will be to see how accurate the "second-order" predictions for these first- and second-order statistics of the fields are, and whether such statistics can be used to gain a priori insight into the actual distribution of the fields in the physical space. More generally, the full distributions of the fields will be computed in order to assess the relative importance of the higher moments (higher than second) of the fields, as well as their implication for the homogenization methods based on the use of linear comparison composites.

\section{Preliminaries on viscoplastic composites}

We consider composite materials made of $N$ different homogeneous constituents, or phases, which are assumed to be randomly distributed in a specimen occupying a volume $\Omega$, 
with boundary $\partial \Omega$, at a length scale that is much smaller than the size of $\Omega$ and the scale of variation of the loading conditions. The constitutive behavior of the viscoplastic phases is characterized by convex dissipation (or strain-rate) potentials $w^{(r)}(r=1, \ldots, N)$, such that the Cauchy stress $\boldsymbol{\sigma}$ and Eulerian strain rate $\boldsymbol{\varepsilon}$ are related by

$$
\boldsymbol{\sigma}=\frac{\partial w}{\partial \boldsymbol{\varepsilon}}(\mathbf{x}, \boldsymbol{\varepsilon}), \quad w(\mathbf{x}, \boldsymbol{\varepsilon})=\sum_{r=1}^{N} \chi^{(r)}(\mathbf{x}) w^{(r)}(\boldsymbol{\varepsilon}),
$$

where the characteristic functions $\chi^{(r)}$ serve to describe the microstructure, being 1 if the position vector $\mathbf{x}$ is in phase $r$, and 0 otherwise.

Let $\langle\cdot\rangle$ and $\langle\cdot\rangle^{(r)}$ denote the volume averages over the composite $(\Omega)$ and over phase $r$ $\left(\Omega^{(r)}\right)$, respectively. We are concerned with the problem of finding the effective behavior of the composite, which is defined as the relation between the average stress $\overline{\boldsymbol{\sigma}}=\langle\boldsymbol{\sigma}\rangle$ and the average strain rate $\overline{\boldsymbol{\varepsilon}}=\langle\boldsymbol{\varepsilon}\rangle$. The effective behavior can be determined from the effective strain-rate potential $\widetilde{W}$, such that

$$
\overline{\boldsymbol{\sigma}}=\frac{\partial \widetilde{W}}{\partial \overline{\boldsymbol{\varepsilon}}}(\overline{\boldsymbol{\varepsilon}}), \quad \widetilde{W}(\overline{\boldsymbol{\varepsilon}})=\inf _{\boldsymbol{\varepsilon} \in \mathscr{K}(\overline{\boldsymbol{\varepsilon}})}\langle w(\mathbf{x}, \boldsymbol{\varepsilon})\rangle=\inf _{\boldsymbol{\varepsilon} \in \mathscr{K}(\overline{\boldsymbol{\varepsilon}})} \sum_{r=1}^{N} c^{(r)}\left\langle w^{(r)}(\boldsymbol{\varepsilon})\right\rangle^{(r)},
$$

where $c^{(r)}$ denotes the volume fraction of phase $r$, and $\mathscr{K}(\overline{\boldsymbol{\varepsilon}})$ is the set of kinematically admissible strain-rate fields, such that there is a velocity field $\mathbf{v}$ satisfying $\varepsilon=[\nabla \mathbf{v}+$ $\left.(\nabla \mathbf{v})^{\mathrm{T}}\right] / 2$ in $\Omega$, and $\mathbf{v}=\overline{\boldsymbol{\varepsilon}} \mathbf{x}$ on $\partial \Omega$. The above formula can be simplified for periodic microstructures, as discussed in more detail in Section 4.1. Physically, $\widetilde{W}$ corresponds to the energy dissipated in the composite when subjected to affine velocities on the boundary, with prescribed average strain rate $\overline{\boldsymbol{\varepsilon}}=\langle\boldsymbol{\varepsilon}\rangle$.

Alternatively, the behavior of the phases can be characterized by convex stress potentials $u^{(r)}$, which are the Legendre transforms of $w^{(r)}$, that is, $u^{(r)}(\boldsymbol{\sigma})=\left(w^{(r)}\right)^{*}(\boldsymbol{\sigma})$. Then, the local stress and strain-rate tensors are related by

$$
\boldsymbol{\varepsilon}=\frac{\partial u}{\partial \boldsymbol{\sigma}}(\boldsymbol{\sigma}), \quad u(\mathbf{x}, \boldsymbol{\sigma})=\sum_{r=1}^{N} \chi^{(r)}(\mathbf{x}) u^{(r)}(\boldsymbol{\sigma})
$$

and the effective behavior can be described in terms of the effective stress potential $\widetilde{U}$, such that

$$
\overline{\overline{\boldsymbol{\varepsilon}}}=\frac{\partial \widetilde{U}}{\partial \overline{\boldsymbol{\sigma}}}(\overline{\boldsymbol{\sigma}}), \quad \widetilde{U}(\overline{\boldsymbol{\sigma}})=\inf _{\boldsymbol{\sigma} \in \mathscr{S}(\overline{\boldsymbol{\sigma}})}\langle u(\mathbf{x}, \boldsymbol{\sigma})\rangle=\inf _{\boldsymbol{\sigma} \in \mathscr{S}(\overline{\boldsymbol{\sigma}})} \sum_{r=1}^{N} c^{(r)}\left\langle u^{(r)}(\boldsymbol{\sigma})\right\rangle^{(r)},
$$

where $\mathscr{S}(\overline{\boldsymbol{\sigma}})$ is the set of divergence-free stresses such that $\overline{\boldsymbol{\sigma}}=\langle\boldsymbol{\sigma}\rangle$.

The variational formulations (2) and (4) can be shown to be completely equivalent, in the sense that the functions $\widetilde{W}$ and $\widetilde{U}$ are Legendre duals of each other. In general, these functions are very difficult to compute, since they require the solution to sets of nonlinear partial differential equations with randomly oscillating coefficients. In the next section we describe a variational method for estimating these effective potentials.

\section{Second-order variational estimates}

In this section, an outline is given of the "second-order" homogenization method introduced by Ponte Castañeda (2002a). Like earlier nonlinear homogenization methods, 
it is based on the construction of a linear comparison composite (LCC) whose constituent phases are identified with appropriate linearizations of the given nonlinear phases. This allows the use of the many different methods already available to bound and estimate the effective behavior of linear composites to generate corresponding estimates for the effective behavior and field statistics in nonlinear heterogeneous media.

\subsection{Estimates for the effective behavior}

The second-order method can be formulated using either strain-rate or stress potentials. We begin here by considering the stress formulation. In this case, an LCC is introduced, with the same microstructure as the nonlinear composite, and with stress phase potentials given by second-order Taylor approximations to the corresponding stress potentials $u^{(r)}$, namely,

$$
u_{T}^{(r)}(\boldsymbol{\sigma})=u^{(r)}\left(\check{\boldsymbol{\sigma}}^{(r)}\right)+\frac{\partial u^{(r)}}{\partial \boldsymbol{\sigma}}\left(\check{\boldsymbol{\sigma}}^{(r)}\right) \cdot\left(\boldsymbol{\sigma}-\check{\boldsymbol{\sigma}}^{(r)}\right)+\frac{1}{2}\left(\boldsymbol{\sigma}-\check{\boldsymbol{\sigma}}^{(r)}\right) \cdot \mathbf{M}^{(r)}\left(\boldsymbol{\sigma}-\check{\boldsymbol{\sigma}}^{(r)}\right),
$$

where $\check{\boldsymbol{\sigma}}^{(r)}$ are uniform reference stresses, and $\mathbf{M}^{(r)}$ are uniform, symmetric, fourth-order tensors (viscous compliances). Note that the stress-strain-rate relations associated with Eq. (5) correspond to that of a "thermoelastic" material, given by $\boldsymbol{\varepsilon}=\mathbf{M}^{(r)} \boldsymbol{\sigma}+\boldsymbol{\eta}^{(r)}$, where $\boldsymbol{\eta}^{(r)}=\partial u^{(r)} / \partial \boldsymbol{\sigma}\left(\check{\boldsymbol{\sigma}}^{(r)}\right)-\mathbf{M}^{(r)} \check{\boldsymbol{\sigma}}^{(r)}$ are uniform strain-rate polarization tensors.

The central idea of the method is to choose the variables $\check{\boldsymbol{\sigma}}^{(r)}$ and $\mathbf{M}^{(r)}$ in such a way as to generate the best possible estimates for the effective potential $\widetilde{U}$ of the nonlinear composite in terms of corresponding estimates for the effective potential $\widetilde{U}_{T}$ of the (thermoelastic) LCC. To that end, a suitably designed variational principle is used, which leads to the following estimate for $\widetilde{U}$ (see Ponte Castañeda, 2002a):

$$
\widetilde{U}(\overline{\boldsymbol{\sigma}})=\underset{\mathbf{M}^{(s)}}{\operatorname{stat}}\left\{\widetilde{U}_{T}\left(\overline{\boldsymbol{\sigma}} ; \check{\boldsymbol{\sigma}}^{(s)}, \mathbf{M}^{(s)}\right)-\sum_{r=1}^{N} c^{(r)} V^{(r)}\left(\check{\boldsymbol{\sigma}}^{(r)}, \mathbf{M}^{(r)}\right)\right\},
$$

where an equality has been used in the sense of a variational approximation, and where the "error functions" $V^{(r)}$ are defined by

$$
V^{(r)}\left(\check{\boldsymbol{\sigma}}^{(r)}, \mathbf{M}^{(r)}\right)=\operatorname{stat}_{\hat{\boldsymbol{\sigma}}^{(r)}}\left\{u_{T}^{(r)}\left(\hat{\boldsymbol{\sigma}}^{(r)}\right)-u^{(r)}\left(\hat{\boldsymbol{\sigma}}^{(r)}\right)\right\} .
$$

The stationary operation in (7) leads to the following conditions:

$$
\frac{\partial u^{(r)}}{\partial \boldsymbol{\sigma}}\left(\hat{\boldsymbol{\sigma}}^{(r)}\right)-\frac{\partial u^{(r)}}{\partial \boldsymbol{\sigma}}\left(\check{\boldsymbol{\sigma}}^{(r)}\right)=\mathbf{M}^{(r)}\left(\hat{\boldsymbol{\sigma}}^{(r)}-\check{\boldsymbol{\sigma}}^{(r)}\right) .
$$

In turn, the stationary operation with respect to the tensors $\mathbf{M}^{(r)}$ in expression (6) leads to the conditions

$$
\left(\hat{\boldsymbol{\sigma}}^{(r)}-\check{\boldsymbol{\sigma}}^{(r)}\right) \otimes\left(\hat{\boldsymbol{\sigma}}^{(r)}-\check{\boldsymbol{\sigma}}^{(r)}\right)=\mathbf{C}_{\boldsymbol{\sigma}}^{(r)}+\left(\overline{\boldsymbol{\sigma}}^{(r)}-\check{\boldsymbol{\sigma}}^{(r)}\right) \otimes\left(\overline{\boldsymbol{\sigma}}^{(r)}-\check{\boldsymbol{\sigma}}^{(r)}\right),
$$

where $\overline{\boldsymbol{\sigma}}^{(r)}=\langle\boldsymbol{\sigma}\rangle^{(r)}$ is the average of the stress over phase $r$, and

$$
\mathbf{C}_{\boldsymbol{\sigma}}^{(r)} \doteq\left\langle\left(\boldsymbol{\sigma}-\overline{\boldsymbol{\sigma}}^{(r)}\right) \otimes\left(\boldsymbol{\sigma}-\overline{\boldsymbol{\sigma}}^{(r)}\right)\right\rangle^{(r)}
$$

denotes the covariance tensor of the stress fluctuations in phase $r$ (Bobeth and Diener, 1987; Parton and Buryachenko, 1990), in the LCC. It should be emphasized that the fourth-order tensor appearing on the left-hand side of Eq. (9) is of rank one, whereas the 
right-hand side is, in general, of full rank. Thus equality cannot be enforced for all components of this tensorial relation, and only certain traces of it have to be used, depending on the form of the tensors $\mathbf{M}^{(r)}$.

From relations (8) and (9), it is seen, as depicted in Fig. 1, that the viscous-compliance tensor $\mathbf{M}^{(r)}$ corresponds to a "generalized secant" linearization of the nonlinear stress-strain-rate relation for phase $r$, which depends on the second-moments of the (intraphase) fluctuations of the stress field.

Finally, using the fact that (6) and (7) are stationary with respect to the tensors $\mathbf{M}^{(r)}$ and $\hat{\boldsymbol{\sigma}}^{(r)}$, respectively, the estimate (6) can be shown to reduce to

$$
\widetilde{U}(\overline{\boldsymbol{\sigma}})=\sum_{r=1}^{N} c^{(r)}\left[u^{(r)}\left(\hat{\boldsymbol{\sigma}}^{(r)}\right)-\frac{\partial u^{(r)}}{\partial \boldsymbol{\sigma}}\left(\check{\boldsymbol{\sigma}}^{(r)}\right) \cdot\left(\hat{\boldsymbol{\sigma}}^{(r)}-\overline{\boldsymbol{\sigma}}^{(r)}\right)\right],
$$

where the reference tensors $\check{\boldsymbol{\sigma}}^{(r)}$ remain to be specified. Ponte Castañeda (2002a) initially suggested that the best possible estimate for $\widetilde{U}$ within this scheme could be obtained by enforcing stationarity of (6) with respect to the tensors $\check{\boldsymbol{\sigma}}^{(r)}$. Unfortunately, it has not yet been possible to satisfy the resulting conditions on $\check{\boldsymbol{\sigma}}^{(r)}$. Because of this, several different prescriptions have been proposed (Ponte Castañeda, 2002b; Idiart and Ponte Castañeda, 2003 , among others). In this work, use will be made of the simple prescription

$$
\check{\boldsymbol{\sigma}}^{(r)}=\overline{\boldsymbol{\sigma}},
$$

proposed recently by Idiart and Ponte Castañeda (2005), although it should be emphasized that the optimal choice of the reference tensors $\check{\boldsymbol{\sigma}}^{(r)}$ remains an open question.

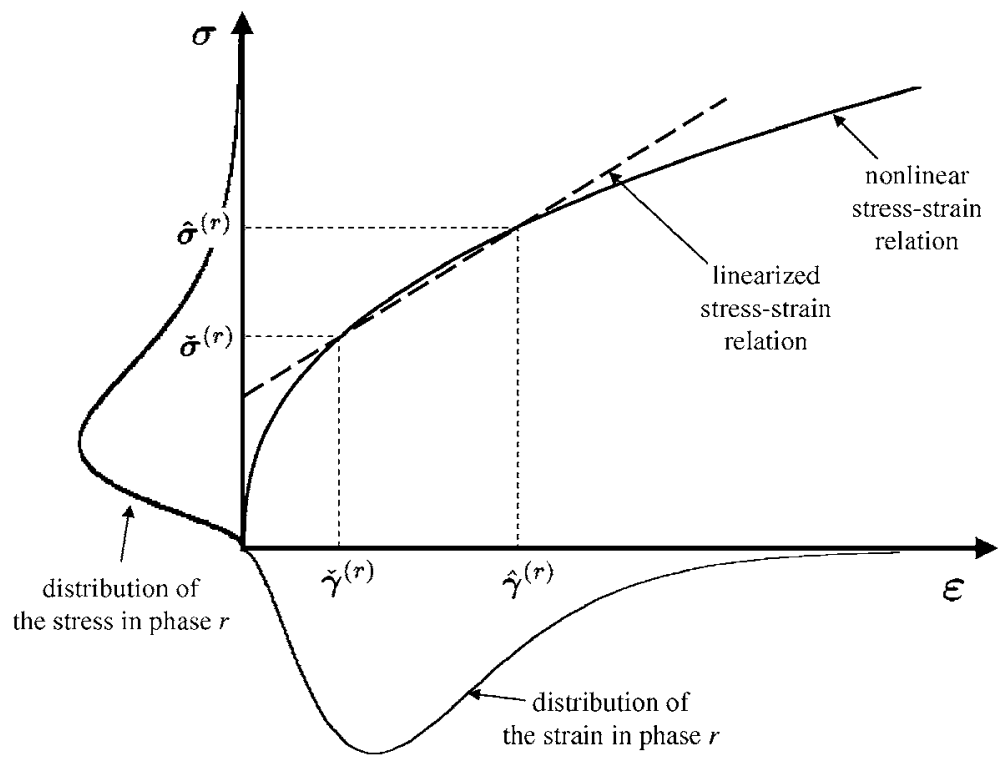

Fig. 1. One-dimensional sketch of the nonlinear stress-strain-rate relation and the "generalized secant" linearization used by the second-order method. The symbols $\check{\gamma}^{(r)}$ and $\hat{\gamma}^{(r)}$ denote $\partial u^{(r)} / \partial \boldsymbol{\sigma}\left(\check{\boldsymbol{\sigma}}^{(r)}\right)$ and $\partial u^{(r)} / \partial \boldsymbol{\sigma}\left(\hat{\boldsymbol{\sigma}}^{(r)}\right)$, respectively. 
Thus, estimate (11) requires the computation of the effective potential $\widetilde{U}_{T}$, which can be obtained using any linear homogenization method appropriate for composites with local potentials $u_{T}^{(r)}$ and the same microstructure as the nonlinear composite. Then, relations (8) and (9) become a system of algebraic nonlinear equations for the variables $\hat{\boldsymbol{\sigma}}^{(r)}$ and $\mathbf{M}^{(r)}$ in each phase, where the tensors $\overline{\boldsymbol{\sigma}}^{(r)}$ and $\mathbf{C}_{\boldsymbol{\sigma}}^{(r)}$ can be computed from the LCC (see expressions (15)-(16)), and expression (11) gives the desired estimate for the effective potential of the nonlinear composite.

An estimate for the dissipation potential $\widetilde{W}$ may be obtained similarly by making use of an LCC with phase potentials $w_{T}^{(r)}$, given by second-order Taylor approximations to the corresponding nonlinear strain-rate potentials $w^{(r)}$, in terms of uniform reference strain rates $\check{\boldsymbol{\varepsilon}}^{(r)}$ and viscosity tensors $\mathbf{L}^{(r)}$. The resulting estimate for the nonlinear strain-rate potential is given by

$$
\widetilde{W}(\overline{\boldsymbol{\varepsilon}})=\sum_{r=1}^{N} c^{(r)}\left[w^{(r)}\left(\hat{\boldsymbol{\varepsilon}}^{(r)}\right)-\frac{\partial w^{(r)}}{\partial \boldsymbol{\varepsilon}}\left(\breve{\boldsymbol{\varepsilon}}^{(r)}\right) \cdot\left(\hat{\boldsymbol{\varepsilon}}^{(r)}-\overline{\boldsymbol{\varepsilon}}^{(r)}\right)\right],
$$

where the tensors $\hat{\boldsymbol{\varepsilon}}^{(r)}$ and $\mathbf{L}^{(r)}$ are determined by expressions analogous to (8) and (9). On the other hand, for reasons that will become evident in the next subsection, the reference strains $\breve{\boldsymbol{\varepsilon}}^{(r)}$ will be chosen in this work such that the estimates (11) and (13) be Legendre duals of each other. One way to ensure this is to set all reference strain rates $\breve{\boldsymbol{\varepsilon}}^{(r)}$ equal to a second-order tensor $\check{\boldsymbol{\varepsilon}}$, and then determine this tensor using the duality condition

$$
\widetilde{U}(\overline{\boldsymbol{\sigma}} ; \check{\boldsymbol{\sigma}})=\widetilde{W}^{*}(\overline{\boldsymbol{\sigma}} ; \check{\boldsymbol{\varepsilon}}) \text { or equivalently } \widetilde{W}(\overline{\boldsymbol{\varepsilon}} ; \check{\boldsymbol{\varepsilon}})=\widetilde{U}^{*}(\overline{\boldsymbol{\varepsilon}} ; \check{\boldsymbol{\sigma}}),
$$

where the superscript * stands for Legendre transform. Thus, the procedure to compute the estimates (13) is similar to that required to compute the estimates (11), but with a different prescription for the tensor $\check{\boldsymbol{\varepsilon}}$ to ensure equivalence of the nonlinear estimates.

\subsection{Statistics of the local fields}

The local fields in the associated LCC constitute an approximation to the local fields in the nonlinear composite. Although such an approximation is not expected to be very accurate in a pointwise sense, it is reasonable to expect that it may yield reasonably accurate estimates for averaged quantities, such as the phase averages and secondmoments of the field fluctuations.

Thus the phase averages and phase covariance tensors of the stress can be obtained from the LCC generated by the stress version, using the following identities (see for example Ponte Castañeda and Suquet, 1998):

$$
\begin{aligned}
& \overline{\boldsymbol{\sigma}}^{(r)}=\mathbf{B}^{(r)} \overline{\boldsymbol{\sigma}}+\mathbf{b}^{(r)}, \\
& \mathbf{C}_{\boldsymbol{\sigma}}^{(r)}=\frac{2}{c^{(r)}} \frac{\partial \widetilde{U}_{T}}{\partial \mathbf{M}^{(r)}}-\left(\overline{\boldsymbol{\sigma}}^{(r)}-\check{\boldsymbol{\sigma}}^{(r)}\right) \otimes\left(\overline{\boldsymbol{\sigma}}^{(r)}-\check{\boldsymbol{\sigma}}^{(r)}\right) .
\end{aligned}
$$

In these expressions, $\mathbf{B}^{(r)}$ and $\mathbf{b}^{(r)}$ are concentration tensors that depend on the tensors $\mathbf{M}^{(r)}$ and $\check{\boldsymbol{\sigma}}^{(r)}$ according to the homogenization procedure utilized, $\widetilde{U}_{T}$ is the effective potential of the LCC, and the derivative should be taken with $\check{\boldsymbol{\sigma}}^{(r)}$ held fixed. Similarly, the phase averages and phase covariance tensors of the strain-rate can be obtained from the LCC generated by the strain-rate version, using analogous expressions. 
It should be remarked at this point that, in general, the LCCs generated by the stress and strain-rate versions are not equivalent to each other, in the sense that $u_{T}^{(r)} \neq\left(w_{T}^{(r)}\right)^{*}$, and therefore the stress quantities (15)-(16) and their strain-rate counterparts correspond to different LCCs. However, the phase averages thus obtained are actually consistent with the stress-strain-rate relation of the nonlinear composite arising from the "second-order" estimates, in the sense that

$$
\overline{\boldsymbol{\varepsilon}}=\frac{\partial \widetilde{U}}{\partial \overline{\boldsymbol{\sigma}}}(\overline{\boldsymbol{\sigma}})=\sum_{r=1}^{N} c^{(r)} \overline{\boldsymbol{\varepsilon}}^{(r)} \quad \text { and } \quad \overline{\boldsymbol{\sigma}}=\frac{\partial \widetilde{W}}{\partial \overline{\boldsymbol{\varepsilon}}}(\overline{\boldsymbol{\varepsilon}})=\sum_{r=1}^{N} c^{(r)} \overline{\boldsymbol{\sigma}}^{(r)},
$$

where $\widetilde{U}$ and $\widetilde{W}$ are the nonlinear estimates (11) and (13). The reason for this is that the LCC associated with the stress (resp. strain-rate) formulation is subjected to the same macroscopic stress (resp. strain-rate) as the nonlinear composite (cf. expression (6)). Then, relations (17) follow from the fact that the effective potentials generated by both versions are Legendre duals of each other, which in turn follows from the choice (14) for the reference strain-rate.

\section{A numerical method based on the fast Fourier transform}

The numerical method used in the present work derives from that initially developed in the context of elasto-plasticity by Moulinec and Suquet (1998), which is based on fast Fourier transforms.

For clarity, the notation used in this section is slightly different from that used in the rest of the paper. The strain rate, denoted by $\varepsilon$ in the rest of the paper, will be denoted here by $\dot{\boldsymbol{\varepsilon}}$, since the constitutive relations which are used for computational purposes involve both the strain $\boldsymbol{\varepsilon}$ and the strain rate $\dot{\boldsymbol{\varepsilon}}$.

\subsection{Elasto-viscoplastic problem}

The Euler equations associated with the variational problem (2) amount to finding a stress field $\boldsymbol{\sigma}$ and a velocity field $\mathbf{v}$ such that

$$
\dot{\boldsymbol{\varepsilon}}=\frac{\partial u}{\partial \boldsymbol{\sigma}}(\mathbf{x}, \boldsymbol{\sigma}), \quad \dot{\boldsymbol{\varepsilon}}=\frac{1}{2}\left(\nabla \mathbf{v}+\nabla \mathbf{v}^{\mathrm{T}}\right), \quad \operatorname{div}(\boldsymbol{\sigma})=0, \quad\langle\dot{\boldsymbol{\varepsilon}}\rangle=\dot{\overline{\boldsymbol{\varepsilon}}}
$$

The problem is closed by imposing periodicity conditions on the boundary of the r.v.e. $V$.

The solution of the nonlinear system (18) is obtained by incorporating elastic effects in the constitutive equations and then taking the limit as the time $t$ tends to $+\infty$ of the solution of an elasto-viscoplastic problem derived from (18) (this procedure is very similar to the approach used by Moulinec and Suquet (1998) to determine the extremal surface of rigid-plastic composites through the resolution of elasto-plastic problems). An alternative method based on augmented Lagrangians has been proposed by Michel et al. (2001). However, in order to get a good convergence rate of this method, a fine tuning of its numerical parameters has to be performed. For the problem considered in the next section, this optimization has to be done for each individual configuration and each nonlinearity exponent. By contrast, the method used in the present study, although slightly slower, is more robust in that it can be used with the same set of parameters for all configurations and all nonlinearity exponents. 
Each nonlinear viscous constituent is given an elasticity characterized by a fourth-order tensor $\mathbf{L}^{(r)}$, and the constitutive relations for the individual constituents now read as

$$
\dot{\boldsymbol{\sigma}}(\mathbf{x})=\mathbf{L}(\mathbf{x})\left(\dot{\boldsymbol{\varepsilon}}(\mathbf{x})-\dot{\boldsymbol{\varepsilon}}^{\mathrm{vp}}(\mathbf{x})\right), \quad \dot{\boldsymbol{\varepsilon}}^{\mathrm{vp}}(\mathbf{x})=\frac{\partial u}{\partial \boldsymbol{\sigma}}(\mathbf{x}, \boldsymbol{\sigma}) .
$$

Eq. (19), complemented by equilibrium and compatibility equations, is solved incrementally. A constant (time-independent) macroscopic strain rate $\dot{\bar{\varepsilon}}$ is applied to the unit-cell, and the time-dependent stress and strain fields (with initial conditions identically 0 ) are determined by a time integration algorithm detailed below. As $t$ tends to $+\infty$ the stress field $\boldsymbol{\sigma}$ and the strain-rate field $\dot{\boldsymbol{\varepsilon}}$ eventually reach an asymptotic state for which $\dot{\boldsymbol{\sigma}}=\mathbf{0}$ and $\dot{\boldsymbol{\varepsilon}}=\dot{\boldsymbol{\varepsilon}}^{\mathrm{vp}}$. These asymptotic fields are solutions of (18). The resolution of the local problem using (19) is numerically simpler and less stiff than that based on (18).

\subsection{Time integration of the constitutive relations}

Relation (19) is an ordinary differential equation expressing the constitutive relations which can be integrated by an implicit step-by step time integration algorithm very similar to the radial-return algorithm used in Moulinec and Suquet (1998).

The time interval $[0, T]$ is discretized into time steps $\left[t^{i}, t^{i+1}\right]$. Assuming that the fields $\boldsymbol{\sigma}^{i}$ and $\boldsymbol{\varepsilon}^{i}$ at time $t=t^{i}$ have been determined, we look for the unknown fields $\boldsymbol{\sigma}^{i+1}$ and $\boldsymbol{\varepsilon}^{i+1}$ at time $t=t^{i+1}$. Replacing time differentiation by a finite difference in (19) gives (the dependence on the phase is omitted for simplicity)

$$
\boldsymbol{\sigma}^{i+1}-\boldsymbol{\sigma}^{i}=\mathbf{L}\left(\boldsymbol{\varepsilon}^{i+1}-\boldsymbol{\varepsilon}^{i}-\left(\dot{\boldsymbol{\varepsilon}}^{\mathrm{vp}}\right)^{i+1}\left(t^{i+1}-t^{i}\right)\right), \quad\left(\dot{\boldsymbol{\varepsilon}}^{\mathrm{vp}}\right)^{i+1}=\frac{\partial u}{\partial \boldsymbol{\sigma}}\left(\boldsymbol{\sigma}^{i+1}\right) .
$$

Assuming that $\boldsymbol{\varepsilon}^{i+1}$ is known, and introducing the elastic "trial" prediction $\boldsymbol{\sigma}_{T}^{i+1}=$ $\boldsymbol{\sigma}^{i}+\mathbf{L}\left(\boldsymbol{\varepsilon}^{i+1}-\boldsymbol{\varepsilon}^{i}\right)$, the nonlinear equation (20) takes the form

$$
\boldsymbol{\sigma}^{i+1}+\left(t^{i+1}-t^{i}\right) \mathbf{L} \frac{\partial u}{\partial \boldsymbol{\sigma}}\left(\boldsymbol{\sigma}^{i+1}\right)=\boldsymbol{\sigma}_{T}^{i+1} .
$$

When the phases are isotropic and incompressible, the nonlinear equation (21) further reduces to a single nonlinear scalar equation. Therefore the solution of (21) can be expressed symbolically as a nonlinear constitutive relation

$$
\boldsymbol{\sigma}^{i+1}(\mathbf{x})=\mathbf{F}^{i+1}\left(\mathbf{x}, \boldsymbol{\varepsilon}^{i+1}(\mathbf{x})\right) \text {. }
$$

In addition, $\boldsymbol{\sigma}^{i+1}$ and $\boldsymbol{\varepsilon}^{i+1}$ must also satisfy the equilibrium and compatibility equations. These partial differential equations coupling different material points in the r.v.e. are treated through a Green's operator approach as in Moulinec and Suquet (1998). More specifically, the solution of the nonlinear local problem

$$
\boldsymbol{\sigma}=\mathbf{F}(\boldsymbol{\varepsilon}), \quad \boldsymbol{\varepsilon}=\frac{1}{2}\left(\nabla \mathbf{u}+\nabla \mathbf{u}^{\mathrm{T}}\right), \quad \operatorname{div}(\boldsymbol{\sigma})=0, \quad\langle\boldsymbol{\varepsilon}\rangle=\overline{\boldsymbol{\varepsilon}},
$$

with periodic boundary conditions, solves the nonlinear integral equation

$$
\varepsilon(\mathbf{u})=-\Gamma^{0} * \delta \mathbf{F}(\boldsymbol{\varepsilon}(\mathbf{u}))+\bar{\varepsilon}, \quad \delta \mathbf{F}(\mathbf{x}, \boldsymbol{\varepsilon})=\mathbf{F}(\mathbf{x}, \boldsymbol{\varepsilon})-\mathbf{L}^{0} \boldsymbol{\varepsilon},
$$

where $\mathbf{L}^{0}$ is the stiffness of a reference medium and $\Gamma^{0}$ the associated Green's operator (see Moulinec and Suquet, 1998). The integral equation (24) is solved by iterations

$$
\boldsymbol{\varepsilon}\left(\mathbf{u}_{k+1}\right)=-\Gamma^{0} * \boldsymbol{\delta} \mathbf{F}\left(\varepsilon\left(\mathbf{u}_{k}\right)\right)+\overline{\boldsymbol{\varepsilon}},
$$


which can be further simplified by noting that $\boldsymbol{\Gamma}^{0} *\left(\mathbf{L}^{0} \boldsymbol{\varepsilon}(\mathbf{u})\right)=\boldsymbol{\varepsilon}(\mathbf{u})-\overline{\boldsymbol{\varepsilon}}$. The final iterative scheme reads

$$
\boldsymbol{\varepsilon}\left(\mathbf{u}_{k+1}\right)=\boldsymbol{\varepsilon}\left(\mathbf{u}_{k}\right)-\Gamma^{0} * \boldsymbol{\sigma}_{k} \quad \text { where } \boldsymbol{\sigma}_{k}=\mathbf{F}\left(\boldsymbol{\varepsilon}\left(\mathbf{u}_{k}\right)\right) .
$$

Finally, this iterative scheme is used with $\mathbf{F}=\mathbf{F}^{i}$ as defined by (22).

\section{Two-phase, power-law, fiber composites}

In what follows, the focus will be on two-phase, fiber composites with random microstructures exhibiting overall transversely isotropic symmetry. The fibers are assumed to be aligned with the $x_{3}$ axis, and will be identified with phase 2 , whereas the continuous phase, called the matrix, will be identified with phase 1 . The individual phases are assumed to be isotropic, incompressible, viscoplastic materials with a constitutive behavior characterized by power-law potentials

$$
w^{(r)}(\boldsymbol{\varepsilon})=\frac{\varepsilon_{0} \sigma_{0}^{(r)}}{1+m}\left(\frac{\varepsilon_{\mathrm{e}}}{\varepsilon_{0}}\right)^{1+m}, \quad u^{(r)}(\boldsymbol{\sigma})=\frac{\varepsilon_{0} \sigma_{0}^{(r)}}{1+n}\left(\frac{\sigma_{\mathrm{e}}}{\sigma_{0}^{(r)}}\right)^{1+n},
$$

where $\varepsilon_{0}$ is a reference strain rate, $m$ is the strain-rate sensitivity, such that $n=1 / m$ and $0 \leqslant m \leqslant 1, \sigma_{0}^{(r)}$ is the flow stress of phase $r$, and the von Mises equivalent strain rate and stress are, respectively, given in terms of the deviatoric strain-rate and stress tensors by $\varepsilon_{\mathrm{e}}=\sqrt{(2 / 3) \boldsymbol{\varepsilon}_{d} \cdot \boldsymbol{\varepsilon}_{d}}$ and $\sigma_{\mathrm{e}}=\sqrt{(3 / 2) \boldsymbol{\sigma}_{d} \cdot \boldsymbol{\sigma}_{d}}$. Note that the limiting values, $m=1$ and $m=0$, correspond to linearly viscous and rigid-perfectly plastic (rate-insensitive) behaviors, respectively.

For simplicity, both phases are assumed to have the same exponent $m$ and reference strain rate $\varepsilon_{0}$. Then, from the homogeneity of the local potentials (27), and the fact that the composites are transversely isotropic, it follows that, under isochoric plane-strain conditions, the effective potentials can be written as

$$
\widetilde{W}(\overline{\boldsymbol{\varepsilon}})=\frac{\varepsilon_{0} \widetilde{\sigma}_{0}}{1+m}\left(\frac{\bar{\varepsilon}_{\mathrm{e}}}{\varepsilon_{0}}\right)^{1+m}, \quad \widetilde{U}(\overline{\boldsymbol{\sigma}})=\frac{\varepsilon_{0} \widetilde{\sigma}_{0}}{1+n}\left(\frac{\bar{\sigma}_{\mathrm{e}}}{\widetilde{\sigma}_{0}}\right)^{1+n},
$$

where $\widetilde{\sigma}_{0}$ is the effective flow stress of the composite, and $\bar{\varepsilon}_{\mathrm{e}}$ and $\bar{\sigma}_{\mathrm{e}}$ are the equivalent macroscopic strain rate and stress, respectively, which are given by $\bar{\varepsilon}_{\mathrm{e}}=(2 / \sqrt{3}) \sqrt{\bar{\varepsilon}_{12}^{2}+}$ $\frac{1}{4}\left(\bar{\varepsilon}_{11}-\bar{\varepsilon}_{22}\right)^{2}$ and $\bar{\sigma}_{\mathrm{e}}=(\sqrt{3}) \sqrt{\bar{\sigma}_{12}^{2}+\frac{1}{4}\left(\bar{\sigma}_{11}-\bar{\sigma}_{22}\right)^{2}}$. This is a very special class of nonlinear (2D) composites, for which the analytical form of the effective potentials is known a priori. The effective behavior is thus completely characterized by $\widetilde{\sigma}_{0}$, which is a function of the strain-rate sensitivity, the heterogeneity contrast, and the concentration of fibers. It should be emphasized, however, that the methods presented above can account for very general microstructures and constitutive behaviors, and that the choice made in this work is dictated by convenience, while preserving the capability of dealing with strongly nonlinear behavior.

It can also be shown for this particular class of nonlinear composites that the local stress and strain-rate fields are homogeneous functions of degree 1 in $\bar{\sigma}_{\mathrm{e}}$ and $\bar{\varepsilon}_{\mathrm{e}}$, respectively. In addition, since the phases and their distribution are isotropic, it is expected that the phase averages are co-axial with the macroscopic averages. It is also expected that the phase 
covariance tensors are "aligned" with the macroscopic averages, in the sense that one of their eigentensors is co-axial with $\overline{\boldsymbol{\sigma}}$ and $\overline{\boldsymbol{\varepsilon}}$. (Indeed, this turns out to be the case in the calculations to follow.) Under incompressible plane-strain conditions, the local stress and strain deviator fields are vectorial in character, thus co-axiality implies proportionality, and so their phase averages can be written as

$$
\overline{\boldsymbol{\sigma}}_{d}^{(r)}=\frac{\bar{\sigma}_{\mathrm{e}}^{(r)}}{\bar{\sigma}_{\mathrm{e}}} \overline{\boldsymbol{\sigma}}_{d} \quad \text { and } \quad \overline{\boldsymbol{\varepsilon}}_{d}^{(r)}=\frac{\bar{\varepsilon}_{\mathrm{e}}^{(r)}}{\bar{\varepsilon}_{\mathrm{e}}} \overline{\boldsymbol{\varepsilon}}_{d},
$$

where the ratios $\bar{\sigma}_{\mathrm{e}}{ }^{(r)} / \bar{\sigma}_{\mathrm{e}}$ and $\bar{\varepsilon}_{\mathrm{e}}{ }^{(r)} / \bar{\varepsilon}_{\mathrm{e}}$ depend only on material parameters. It is also natural to identify two "components" of the strain-rate (resp. stress) tensor which represent its projections "parallel", $\varepsilon_{\|}\left(\right.$resp. $\left.\sigma_{\|}\right)$, and "perpendicular", $\varepsilon_{\perp}\left(\right.$ resp. $\left.\sigma_{\perp}\right)$, to the macroscopic strain rate (resp. stress). These components can be determined (up to a sign) by the two orthogonal fourth-order projection tensors $\mathbf{E}$ and $\mathbf{F}$ given by expressions (56) in Ponte Castañeda (2002a), with $\check{\boldsymbol{\sigma}}^{(r)}=\overline{\boldsymbol{\sigma}}$, through the following relations: $\varepsilon_{\|}^{2}=(2 / 3)(\boldsymbol{\varepsilon} \cdot \mathbf{E} \boldsymbol{\varepsilon})$, $\varepsilon_{\perp}^{2}=(2 / 3)(\boldsymbol{\varepsilon} \cdot \mathbf{F} \boldsymbol{\varepsilon}), \sigma_{\|}^{2}=(3 / 2)(\boldsymbol{\sigma} \cdot \mathbf{E} \boldsymbol{\sigma})$, and $\sigma_{\perp}^{2}=(3 / 2)(\boldsymbol{\sigma} \cdot \mathbf{F} \boldsymbol{\sigma})$. They are such that $\varepsilon_{\mathrm{e}}^{2}=$ $\varepsilon_{\|}^{2}+\varepsilon_{\perp}^{2}$ and $\sigma_{\mathrm{e}}^{2}=\sigma_{\|}^{2}+\sigma_{\perp}^{2}$. For instance, in the numerical simulations to follow, the macroscopic stress will be taken to be

$$
\overline{\boldsymbol{\sigma}}=\bar{\sigma}_{12}\left(\mathbf{e}_{1} \otimes \mathbf{e}_{2}+\mathbf{e}_{2} \otimes \mathbf{e}_{1}\right),
$$

so that the corresponding "parallel" and "perpendicular" components of the local fields are

$$
\sigma_{\|}=\sqrt{3} \sigma_{12}, \quad \sigma_{\perp}=\sqrt{3} \frac{\sigma_{11}-\sigma_{22}}{2}, \quad \varepsilon_{\|}=\frac{2}{\sqrt{3}} \varepsilon_{12}, \quad \varepsilon_{\perp}=\frac{2}{\sqrt{3}} \frac{\varepsilon_{11}-\varepsilon_{22}}{2} .
$$

The standard deviations of the spatial distributions within each phase of the quantities (31) $\left(\operatorname{SD}^{(r)}(\cdot)=\sqrt{\left\langle(\cdot)^{2}\right\rangle^{(r)}-\left(\langle\cdot\rangle^{(r)}\right)^{2}}\right)$ provide a measure of the intraphase field fluctuations, and are given in terms of the phase covariance tensors by

$$
\begin{aligned}
& \operatorname{SD}^{(r)}\left(\sigma_{\|}\right)=\sqrt{\frac{3}{2} \mathbf{E} \cdot \mathbf{C}_{\sigma}^{(r)}}, \quad \operatorname{SD}^{(r)}\left(\sigma_{\perp}\right)=\sqrt{\frac{3}{2} \mathbf{F} \cdot \mathbf{C}_{\boldsymbol{\sigma}}^{(r)}}, \\
& \operatorname{SD}^{(r)}\left(\varepsilon_{\|}\right)=\sqrt{\frac{2}{3} \mathbf{E} \cdot \mathbf{C}_{\varepsilon}^{(r)}}, \quad \operatorname{SD}^{(r)}\left(\varepsilon_{\perp}\right)=\sqrt{\frac{2}{3} \mathbf{F} \cdot \mathbf{C}_{\varepsilon}^{(r)}}
\end{aligned}
$$

From the homogeneity of the local fields in $\bar{\sigma}_{\mathrm{e}}$ and $\bar{\varepsilon}_{\mathrm{e}}$, it follows that the ratios $\operatorname{SD}^{(r)}(\sigma) / \bar{\sigma}_{\mathrm{e}}$ and $\mathrm{SD}^{(r)}(\varepsilon) / \bar{\varepsilon}_{\mathrm{e}}$ depend only on the material parameters.

In this work, a special class of random transversely isotropic microstructures is considered known as the composite cylinder assemblage (CCA), introduced by Hashin and Rosen (1964), in which aligned homothetic composite cylinders of an infinite number of sizes fill the entire space. The interest in this type of microstructures is that, in the linear case, their effective behavior is known to be well approximated by the Hashin-Shtrikman (HS) estimates (Hashin and Shtrikman, 1963), at least when the constituent phases are isotropic. Because of this, the "second-order" estimates for nonlinear composites with this type of microstructures will be generated by making use of the linear HS estimates to determine the homogenized behavior of the associated LCC.

In order to carry out full-field numerical simulations for this class of composites using the FFT method, 20 different configurations of the unit-cell were generated by randomly placing in a square cell self-similar non-overlapping composite cylinders of three different 


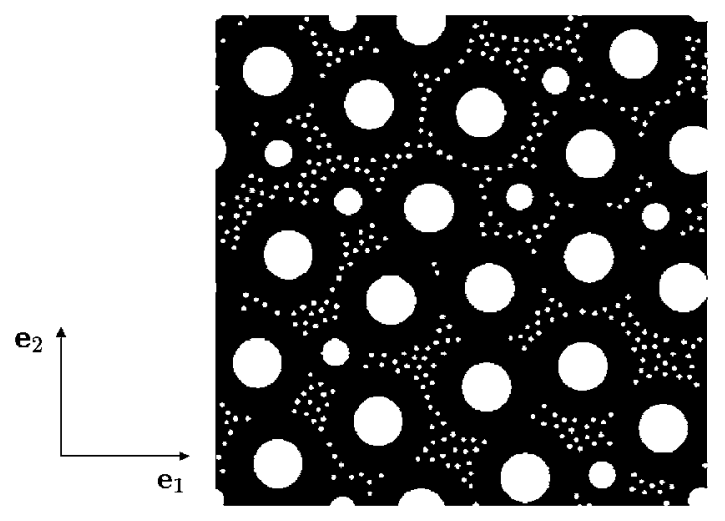

Fig. 2. Typical configuration of the unit cell used in the full-field FFT simulations. It contains 490 composite cylinders of three different sizes, randomly distributed.

sizes, with periodicity conditions for cylinders intersecting the cell boundaries. Fig. 2 shows a typical configuration for the unit cell, containing 490 composite cylinders. Each composite cylinder is composed of a circular core (in white in Fig. 2) surrounded by a circular layer of matrix (in black). It is emphasized that these microstructures constitute an approximation to the CCAs described above, since only a finite number of sizes is used for the composite cylinders. As a consequence of using a finite number of sizes for the composite cylinders, as well as a different number of cylinders of a given size from one configuration to another, the fiber concentration in each configuration is not exactly the same, and ranged between 0.203 and 0.210 , the average value being 0.20626 .

The issues of statistical homogeneity and isotropy for this type of microstructures are briefly discussed in Moulinec and Suquet (2004) (see also Kanit et al., 2003, for a related problem). Both the number of composite cylinders per unit-cell and the number of different configurations used in the analysis result from a compromise between several constraints. First, each configuration has to be large enough to ensure that the periodicity conditions play almost no role on the effective properties. Second, by considering large unit-cells containing a large number of inclusions (several hundreds), the scatter in the quantities of interest (effective properties, first and second moments of the fields) is small and only a few configurations are necessary for the ensemble average of these quantities to reach stationarity.

The FFT results provided in the next section for the effective properties, phase averages, and standard deviations of the local fields are ensemble averages of the computational results over all configurations, and they are taken as approximate values for a fiber concentration of 0.20626 . The way these ensemble averages were performed can be found in Moulinec and Suquet $(2003,2004)$. In addition to the phase averages and covariance tensors, the histograms of the spatial distribution of the local fields can be obtained from the FFT simulations. For any scalar field $z$, it is convenient to introduce a "density of states per unit volume" or "probability density function", $\mathscr{P}_{z}^{(r)}(z)$, defined so that $\mathscr{P}_{z}^{(r)}(z) \mathrm{d} z$ is the volume fraction of phase $r$ in a given configuration where the variable $z$ takes values in the range $z$ and $z+\mathrm{d} z$. (Note that the first and second moments of $z$ are then given by $\left\langle z^{q}\right\rangle^{(r)}=\int_{-\infty}^{\infty} z^{q} \mathscr{P}_{z}^{(r)}(z) \mathrm{d} z$ with $q=1$ and $q=2$, respectively.) At a given value $z_{i}$, the function $\mathscr{P}_{z}^{(r)}$ is computed from the numerical simulations by counting the number of pixels 
$N_{i}^{(r)}$ out of the total number of pixels $N^{(r)}$ in phase $r$ where the variable $z$ takes values between $z_{i} \leqslant z<z_{i}+\Delta z$. In this work, the following relations have been used:

$$
\mathscr{P}_{z}^{(r)}\left(z_{i}\right)=\frac{1}{\Delta z} \frac{N_{i}^{(r)}}{N^{(r)}}, \quad z_{i}=z_{\min }+i \Delta z, \quad \Delta z=\frac{z_{\max }-z_{\min }}{N_{\mathrm{b}}-1},
$$

where $N_{\mathrm{b}}$ denotes the number of bars in the histogram (200 in this work), $z_{\min }$ and $z_{\max }$ denote, respectively, the minimum and maximum values that the quantity $z$ takes in a given simulation, and $i=0,1, \ldots, N_{\mathrm{b}}-2$. For the last bar, $N_{N_{\mathrm{b}}-1}^{(r)}$ is set equal to the number of pixels where $z$ takes the value $z_{\max }$.

The unit-cells used in the numerical simulations were discretized into $1024 \times 1024$ pixels. They were subjected to an in-plane shear stress (30), and the computations were run until the macroscopic strain reached $\bar{\varepsilon}_{12}=0.5$.

\section{Results and discussion}

In this section, the "second-order" (SO) estimates of the Hashin-Shtrikman type, described in the previous section, for the effective behavior and statistics of the local fields in power-law composites are compared with corresponding results generated by FFT fullfield simulations. The results are presented as a function of the strain-rate sensitivity $m$, for a given concentration of fibers $\left(c^{(2)}=0.20626\right)$. Two values of the heterogeneity contrast are considered, one corresponding to fiber-reinforced composites $\left(\sigma_{0}^{(2)} / \sigma_{0}^{(1)}=5\right)$ and the other to fiber-weakened composites $\left(\sigma_{0}^{(2)} / \sigma_{0}^{(1)}=0.2\right)$. Full-field simulations were carried out for several values of the strain-rate sensitivity $(1 / m=n=1,2,3,5,10, \infty)$. The dark circles representing the FFT results in the plots to follow correspond to ensemble averages over 20 different configurations (like the one shown in Fig. 2), and the "error" bars (where given) correspond to the maximum and minimum values, quantifying the scatter of the numerical results.

In addition, comparisons are also provided with the earlier "variational" (VAR) method of Ponte Castañeda (1991) and Suquet (1993), as well as with the "tangent second-order" (TSO) method of Ponte Castañeda (1996), once again, making use of the HS estimates for the relevant LCC. It is recalled here that these methods arise from considering different linearization schemes. Thus, the "variational" method makes use of an LCC whose phases are identified with the "secant" viscous-compliance/viscosity tensors of the nonlinear phases, evaluated at the second-moments of the local fields over the phases (Suquet, 1995; $\mathrm{Hu}, 1996)$. It is emphasized that since the nonlinear phases are isotropic, the corresponding LCC is also locally isotropic in this model. On the other hand, in the earlier version of the "second-order" method (TSO), the phases of the LCC are identified with the "tangent" viscous-compliance/viscosity tensors of the nonlinear phases, evaluated at the phase averages of the local fields. Therefore, unlike the "second-order" method outlined above, this method does not take into account the field fluctuations in the linearization. It should also be mentioned that the TSO estimates are known to have a duality gap (Ponte Castañeda, 1996), and so two sets of estimates corresponding to the strain-rate $(W)$ and stress $(U)$ versions are shown. Finally, the classical estimates of Voigt and Reuss are also included for comparison purposes. Because of this reason, the TSO estimates for strainrate statistics correspond to the $W$ version, while those for the stress statistics correspond to the $U$ version. These are rigorous upper and lower bounds, independent of the 
microstructure, which are obtained by using uniform strain-rate and stress trial fields in the minimum energy principles (2) and (4), respectively.

Before proceeding with the discussion of the results, it is useful to recall that the nonlinear homogenization methods based on the use of an LCC involve two different levels of approximation. The first one consists in the linearization of the behavior of each phase in the nonlinear composite, in order to generate the LCC. Once the LCC is generated, however, its effective behavior needs to be computed. In general, computing exactly the effective behavior of the LCC, which is a random composite, is still a difficult problem, and therefore a second level of approximation is required, which consists in estimating the effective behavior of the LCC, making use of suitable linear homogenization estimates. Therefore, any differences between the homogenization results and the numerical simulations shown below could have either one (or both) of two sources: the estimate used for the LCC (in this case, the HS estimates) or the linearization procedure itself (SO, TSO, VAR).

\subsection{Fiber-weakened composites}

Effective behavior. In Fig. 3a, the various bounds and estimates for the effective flow stress $\widetilde{\sigma}_{0}$ of a fiber-weakened composite, and the corresponding FFT results, are plotted as a function of the strain-rate sensitivity $m$. (Part (b) will be discussed in the next subsection.) The results are normalized by the flow stress of the matrix $\sigma_{0}^{(1)}$. It can be observed that the FFT simulations yield a $\widetilde{\sigma}_{0}$ which decreases slightly with decreasing values of $m$ (i.e., increasing nonlinearity). The main observation, however, is that the SO estimates are found to be in good agreement with the FFT simulations, for weak to moderate nonlinearities $(0.2 \leqslant m \leqslant 1)$, but the agreement is found to deteriorate close to the perfectly plastic limit $(m \rightarrow 0)$, where the FFT results keep decreasing with $m$, while the SO estimates exhibit a slight increase. As explained in more detail below, the reason for such
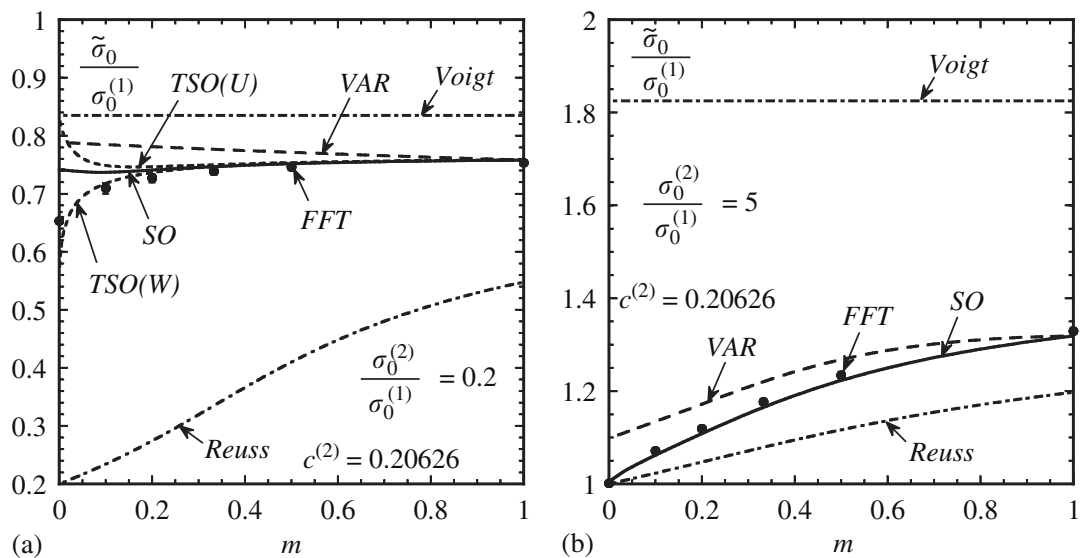

Fig. 3. Effective flow stress $\widetilde{\sigma}_{0}$, normalized by the flow stress of the matrix $\sigma_{0}^{(1)}$, for power-law fiber composites subjected to in-plane shear, as a function of the strain-rate sensitivity $m$, for a given concentration of fibers $\left(c^{(2)}=0.20626\right)$. Comparisons between the "second-order" (SO), "tangent second-order" (TSO), and "variational" (VAR) estimates of the Hashin-Shtrikman type, and the FFT results, for the cases of (a) weaker fibers $\left(\sigma_{0}^{(2)} / \sigma_{0}^{(1)}=0.2\right)$ and (b) stronger fibers $\left(\sigma_{0}^{(2)} / \sigma_{0}^{(1)}=5\right)$. 
differences as $m \rightarrow 0$ could be related to the use of HS estimates for the LCC. It is worth noting though that for $m=0.1$ the differences are still relatively small (approx. 3.5\%). Also included in the figure are both versions of the TSO estimates, as well as the "variational" estimates. The latter are known to provide rigorous upper bounds for the $\widetilde{\sigma}_{0}$ of the class of composites here considered (see for example, Ponte Castañeda and Suquet, 1998). Several observations are in order. First, the homogenization estimates all coincide for $m=1$ with the linear HS estimate, as they should, but give diverging predictions as $m$ decreases. However, it is seen that the TSO and SO estimates give very close predictions for weak to moderate nonlinearities $(0.3 \leqslant m \leqslant 1)$, and it is only for strong nonlinearities that they differ significantly. In this connection, it is noted that, as already pointed out by Ponte Castañeda (2002b), the TSO estimates exhibit a large duality gap for small values of $m$, and in the limit $m \rightarrow 0$ the $\operatorname{TSO}(U)$ estimates tend to the Voigt bound, thus violating the sharper "variational"upper bound. In contrast, the new SO estimates have no duality gap, and are found to satisfy the "variational" upper bound for all values of $m$. Finally, it is noted that the scatter exhibited by the FFT results, although barely noticeable in this figure, increases with increasing nonlinearity. This scatter is due to several reasons, such as the finite size of the specimens, as well as the fact that all specimens have slightly different fiber concentrations. In any event, the scatter is found to be very small even for the smaller values of $m$, suggesting that the quality of the numerical results is good.

Statistics of the local fields. Corresponding estimates for the phase averages and standard deviations of the stress field are given in Fig. 4. In part (a), the equivalent average stresses in each phase $\bar{\sigma}_{\mathrm{e}}{ }^{(r)}$ are shown, normalized by the equivalent macroscopic stress $\bar{\sigma}_{\mathrm{e}}$. It can be seen that all the homogenization estimates are in good agreement with the FFT simulations, for all values of $m$. This is perhaps not surprising, since for the extreme case of void fibers (i.e., $\sigma_{0}^{(2)}=0$ ) all these methods give the exact result (i.e., $\bar{\sigma}_{\mathrm{e}}^{(1)} / \bar{\sigma}_{\mathrm{e}}=1 / c^{(1)}$ and $\left.\bar{\sigma}_{\mathrm{e}}{ }^{(2)} / \bar{\sigma}_{\mathrm{e}}=0\right)$. Nonetheless, it is worth mentioning that the SO estimates are the most consistent ones with the FFT results, the agreement being excellent. Part (b) shows the standard deviations of the stress field in the matrix, as given by expressions (32), normalized by the equivalent macroscopic stress $\bar{\sigma}_{\mathrm{e}}$. The main observation in the context of
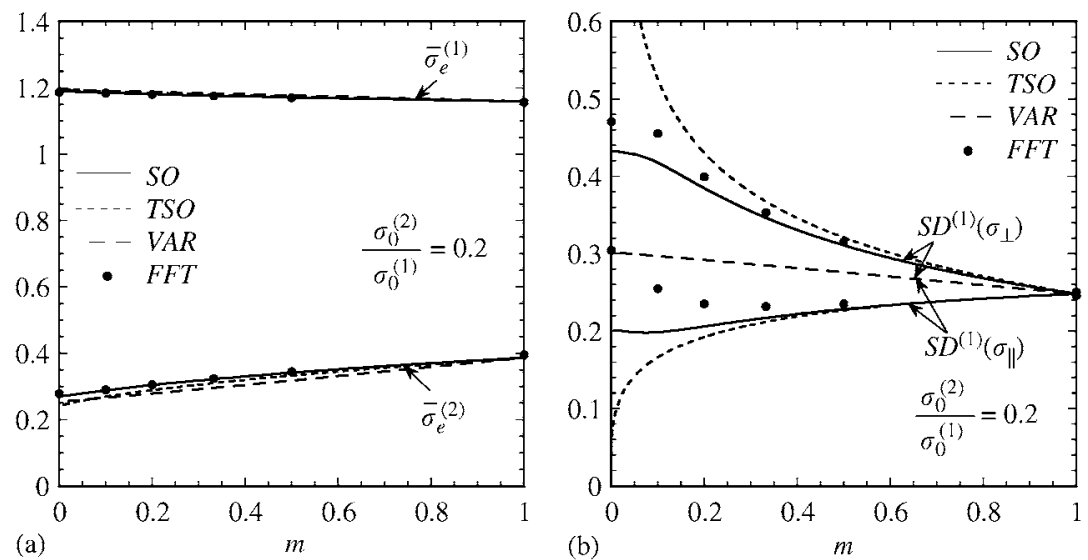

Fig. 4. Statistics of the stress field for the case of weaker fibers. (a) Equivalent average stresses in the matrix $\left(\bar{\sigma}_{\mathrm{e}}{ }^{(1)}\right)$ and in the fibers $\left(\bar{\sigma}_{\mathrm{e}}^{(2)}\right)$. (b) Standard deviation (SD) of the "parallel" and "perpendicular" components of the stress field in the matrix. The results are normalized by the equivalent macroscopic stress $\bar{\sigma}_{\mathrm{e}}$. 
this figure is that the FFT simulations show that, while the stress fluctuations are isotropic (i.e., $\left.\operatorname{SD}^{(1)}\left(\sigma_{\|}\right)=\operatorname{SD}^{(1)}\left(\sigma_{\perp}\right)\right)$ in the linear case, they become progressively more anisotropic as the nonlinearity increases, being larger for the "perpendicular" component than for the "parallel" component. Furthermore, the "perpendicular" fluctuations are found to increase with decreasing $m$, while the "parallel" fluctuations exhibit a non-monotonic dependence on $m$. It can be seen in the figure that the SO estimates are consistent with these observations, exhibiting good agreement with the FFT results for weak to moderate nonlinearities $(0.3 \leqslant m \leqslant 1)$. However, this agreement is seen to deteriorate close to the perfectly plastic limit, where the SO estimates underestimate the stress fluctuations. On the other hand, while for weak to moderate nonlinearities the $\operatorname{TSO}(U)$ estimates are very similar to the SO estimates, as $m \rightarrow 0$ they give vanishing and infinite fluctuations in the "parallel" and "perpendicular" directions, respectively, which is in disagreement with the FFT results. Finally, the "variational" estimates are seen to predict isotropic stress fluctuations for all values of $m$, and are thus inconsistent with the FFT simulations.

Fig. 5 provides corresponding estimates for the phase averages and standard deviations of the strain-rate field. Part (a) shows the equivalent average strain rates in each phase $\bar{\varepsilon}_{\mathrm{e}}{ }^{(r)}$, normalized by the equivalent macroscopic strain rate $\bar{\varepsilon}_{\mathrm{e}}$. It can be seen that the FFT simulations yield an average strain rate in the (weaker) fibers which is higher than that in the matrix, as expected, and that the former increases with nonlinearity while the latter decreases. (Note that, in view of relations (29), these quantities are related by $c^{(1)}\left(\bar{\varepsilon}_{\mathrm{e}}^{(1)} / \bar{\varepsilon}_{\mathrm{e}}\right)+c^{(2)}\left(\bar{\varepsilon}_{\mathrm{e}}^{(2)} / \bar{\varepsilon}_{\mathrm{e}}\right)=1$.) Among the homogenization estimates, the SO estimates are found to be, once again, the most consistent with the FFT results, the agreement being very good for all values of $m$. Although the $\operatorname{TSO}(W)$ estimates give very similar predictions in the range $0.2 \leqslant m \leqslant 1$, they are seen to deviate significantly as $m \rightarrow 0$. In fact, in this limit, they predict a vanishing average strain rate in the matrix, which is inconsistent with the FFT results. In this connection, it is worth mentioning that the SO estimates with reference tensors $\check{\boldsymbol{\sigma}}^{(r)}$ identified with the phase averages predict an average strain rate in the matrix that also vanishes as $m \rightarrow 0$ (see Idiart and Ponte Castañeda, 2003, 2005). Thus the
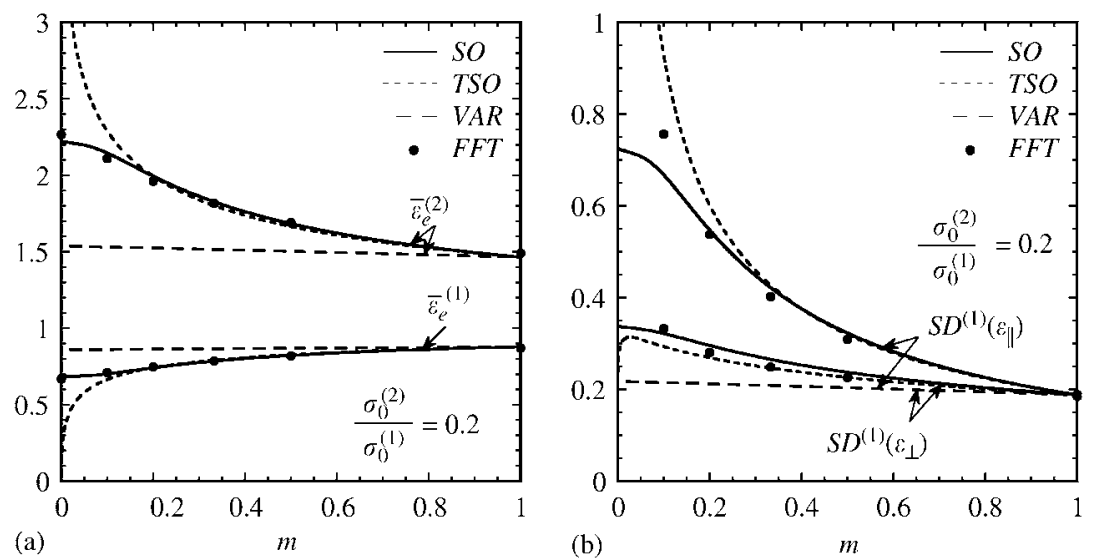

Fig. 5. Statistics of the strain-rate field for the case of weaker fibers. (a) Equivalent average strain rates in the matrix $\left(\bar{\varepsilon}_{\mathrm{e}}^{(1)}\right)$ and fibers $\left(\bar{\varepsilon}_{\mathrm{e}}^{(2)}\right)$. (b) Standard deviation (SD) of the "parallel" and "perpendicular" components of the strain-rate field in the matrix. The results are normalized by the equivalent macroscopic strain rate $\bar{\varepsilon}_{\mathrm{e}}$. 
alternative prescription (12)-(14) used in this work is found to give much more reasonable estimates, especially for strong nonlinearities. Finally, it is observed that the "variational" estimates are inconsistent with the FFT simulations, being almost insensitive to $m$. Part (b) provides plots for the standard deviations of the strain-rate field in the matrix, normalized by the equivalent macroscopic strain rate $\bar{\varepsilon}_{\mathrm{e}}$. The main observation in the context of this figure is that the FFT simulations show that the strain-rate fluctuations, which are isotropic in the linear case, increase significantly and become progressively more anisotropic as the nonlinearity increases. Furthermore, it is observed that, unlike the stress fluctuations (cf. Fig. 4b), the strain-rate fluctuations are larger for the "parallel" component than for the "perpendicular" component. As will be seen in detail shortly, the increase of the strain-rate fluctuations and their anisotropy are consequences of strain localization, which becomes more pronounced with increasing nonlinearity. It can be seen in the figure that the SO estimates are in good agreement with the FFT results for weak to moderate nonlinearities $(0.2 \leqslant m \leqslant 1)$, but they are found to underestimate the strain-rate fluctuations for small values of $m$, yielding finite values in the ideally plastic limit, while the FFT results seem to be consistent with unbounded fluctuations in this limit (the FFT results for $m=0$ yield strain-rate fluctuations that are very large but finite, since the numerical procedure cannot handle infinite quantities). The $\operatorname{TSO}(W)$ estimates are seen to give similar predictions to the SO estimates for weak to moderate nonlinearities, but as $m \rightarrow 0$ they give infinite and vanishing fluctuations for the "parallel" and "perpendicular" components, respectively, which is also inconsistent, since the FFT results suggest that the fluctuations blow up for both components. Finally, the "variational" estimates are seen to be almost insensitive to $m$ here as well, and more importantly, they predict isotropic fluctuations for all values of $m$, which is inconsistent with the FFT simulations.

At this point, it is worth noting that the degree of anisotropy of the stress and strain-rate fluctuations predicted by the different homogenization methods depends strongly on the anisotropy of the compliance/elastic tensors used in the LCC. In fact, for the cases considered here, the compliance/elastic tensors used by these methods are all of the same form, as given by expressions (55) and (44) in Ponte Castañeda (2002a), and it can be verified that the anisotropy of the HS estimates for the stress fluctuations in the matrix is $\mathrm{SD}^{(1)}\left(\sigma_{\|}\right) / \mathrm{SD}^{(1)}\left(\sigma_{\perp}\right)=\sqrt{k}$, while that of the strain-rate fluctuations is $\operatorname{SD}^{(1)}\left(\varepsilon_{\|}\right) /$ $\mathrm{SD}^{(1)}\left(\varepsilon_{\perp}\right)=1 / \sqrt{k}$, where $k=\lambda_{0}^{(1)} / \mu_{0}^{(1)}$ is the (anisotropy) ratio of the "parallel" and "perpendicular" shear moduli. Thus, as already mentioned, the "variational" method makes use of isotropic compliance tensors (when the nonlinear phase is isotropic), so $k=1$, and consequently it predicts isotropic stress and strain-rate fluctuations for all values of $m$. On the other hand, the TSO estimates make use of the "tangent" compliance tensor, which for a power-law phase is of the form mentioned above with $k=m$. This still constitutes a strong restriction, for the anisotropy of the fluctuations is thus given by the strain-rate sensitivity, and cannot depend, for example, on the microstructure. In contrast, the compliance tensors used by the new SO estimates are somewhat more general (see Section 3), allowing for $k$ to depend not only on $m$ but also on the heterogeneity contrast and concentration of fibers. This is one of the reasons why the "second-order" method is able to give superior predictions over the earlier "tangent second-order" and "variational" methods. Finally, it is noted that, the $k$ associated with the $W$ and $U$ versions of the SO estimates are not equal, thus allowing the anisotropy of the strain-rate and stress fluctuations to be different from each other, which is in agreement with the FFT simulations. 


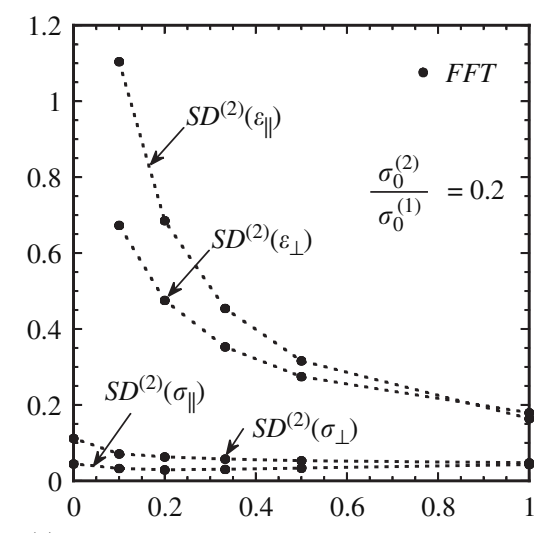

(a)

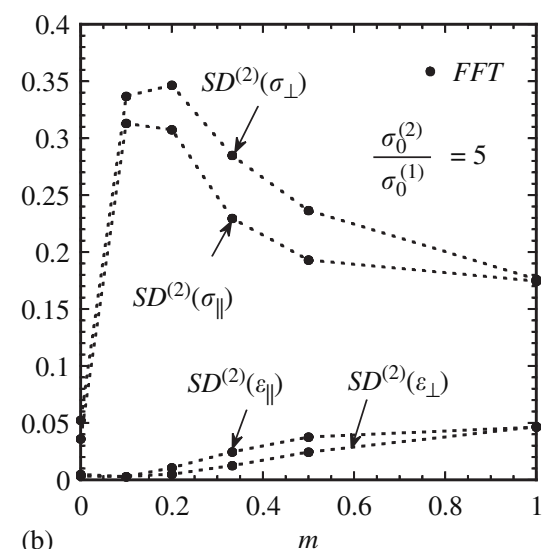

(b)

Fig. 6. FFT results for the standard deviations (SD) of the fields in the fibers, for the cases of (a) weaker fibers $\left(\sigma_{0}^{(2)} / \sigma_{0}^{(1)}=0.2\right)$ and (b) stronger fibers $\left(\sigma_{0}^{(2)} / \sigma_{0}^{(1)}=5\right)$. The SD of the stress components are normalized by the equivalent macroscopic stress $\bar{\sigma}_{\mathrm{e}}$, while the $\mathrm{SD}$ of the strain-rate components are normalized by the equivalent macroscopic strain rate $\bar{\varepsilon}_{\mathrm{e}}$.

The corresponding standard deviations of the local fields in the fiber phase are shown in Fig. 6a. (Part (b) will be discussed in the next subsection.) It can be seen that the FFT results yield stress fluctuations in the fibers that are small, yet finite, in comparison with those in the matrix phase (cf. Fig. 4b), for all values of $m$. On the other hand, the strainrate fluctuations are comparable to those in the matrix phase when $m=1$, and more importantly, they are seen to increase significantly with decreasing $m$, becoming even larger than those in the matrix phase (cf. Fig. 5b). Furthermore, they seem to be consistent with unbounded strain-rate fluctuations in the perfectly plastic limit (the FFT results for $m=0$ yield very large strain-rate fluctuations in this phase as well). Such an increase of the strain fluctuations in the fiber phase is due to the fact that, as the nonlinearity increases, the strain rate also becomes localized in this (weaker) phase, as is discussed further below. In contrast, the nonlinear homogenization estimates provided in this work require zero field fluctuations in the fibers. The reason for this is that use has been made of the Hashin-Shtrikman estimates to homogenize the associated LCCs, which assume that the local fields are uniform and equal inside all the fibers, and this assumption carries over to the nonlinear estimates. It should be emphasized, however, that this is a limitation associated only with the linear HS estimates and not with the nonlinear homogenization methods considered here. The fact that the strain-rate fluctuations in the FFT simulations become very large for small values of $m$ suggests that, for the class of microstructures considered here, the linear HS estimates used in the context of the nonlinear homogenization methods may be inappropriate as $m \rightarrow 0$. In this connection, it is noted that for strong nonlinearities, the LCC generated by the "second-order" method has highly anisotropic phases, and the linear HS estimates, which are known to be accurate for CCA microstructures with isotropic phases, may not give accurate predictions for composites with CCA microstructures when the phases are that anisotropic, at least for the case of weaker fibers. This could explain why the SO estimates and the FFT results provided in this subsection are generally found to be in very good agreement for weak to moderate nonlinearities, while for strong nonlinearities they are found to exhibit different trends. In 
particular, the fact that the SO estimates for the effective flow stress become larger than the corresponding FFT results (see Fig. 3a) is consistent with the fact that the requirement of uniform fields in the fiber phase implicit in the HS hypothesis should lead to a stiffer macroscopic behavior, since, in a sense, it "prevents" the localization of the strain field in the fibers. In turn, this suggests that a better correlation should be obtained by using the "second-order" method in combination with more appropriate linear homogenization methods, or even by computing numerically the homogenized behavior of the LCC, as has been recently done in combination with the "variational" method by Moulinec and Suquet (2004).

Distribution of local fields. For a fuller understanding of the results discussed above, maps of the local fields were generated from the FFT simulations. The maps provided in this work correspond to a composite with the microstructure shown in Fig. 2, subject to inplane shear (30). Thus, in this case the "parallel" and "perpendicular" components of the fields refer to those defined by expressions (31).
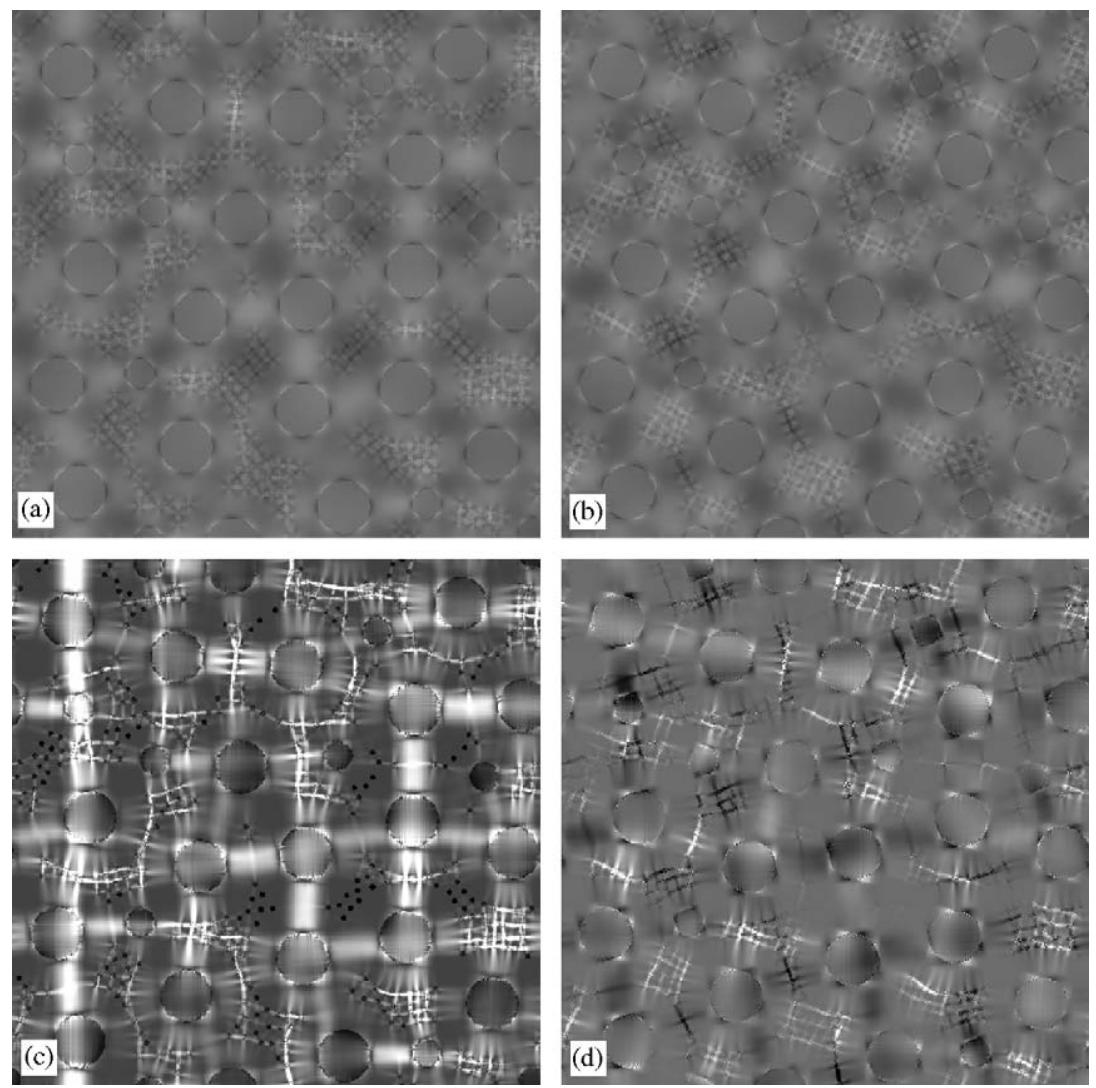

Fig. 7. Strain-rate distribution in a power-law composite with weaker fibers $\left(\sigma_{0}^{(2)} / \sigma_{0}^{(1)}=0.2\right)$, subjected to in-plane shear $\bar{\sigma}_{12}$. The microstructure is the one shown in Fig. 2. Distribution of the "parallel" component $\varepsilon_{12}-\bar{\varepsilon}_{12}^{(r)}$ when (a) $m=1$ and (c) $m=0.1$; distribution of the "perpendicular" component $\left(\varepsilon_{11}-\varepsilon_{22}\right) / 2$ when (b) $m=1$, (d) $m=0.1$. The quantities are normalized by $(\sqrt{3} / 2) \bar{\varepsilon}_{\mathrm{e}}$. Black and white correspond, respectively, to values smaller than -2 and larger than 2 . 
Fig. 7 provides maps of the components of the strain-rate field. Parts (a) and (c) show the deviation in each phase of the "parallel" component about its phase average, that is, $\varepsilon_{12}(\mathbf{x})-\bar{\varepsilon}_{12}^{(1)}$ in the matrix and $\varepsilon_{12}(\mathbf{x})-\bar{\varepsilon}_{12}^{(2)}$ in the fibers, while parts (b) and (d) show the "perpendicular" component $\left(\varepsilon_{11}-\varepsilon_{22}\right) / 2$, which fluctuates about zero in both phases. In turn, parts (a) and (b) correspond to the case of linear $(m=1)$ phases, while parts (c) and (d) correspond to the case of highly nonlinear $(m=0.1)$ phases. These maps show the changing character of the spatial distribution of the strain rate with nonlinearity. Thus, in the linear case, the distributions of both components of the strain rate are rather diffuse and exhibit similar degrees of heterogeneity (see parts (a) and (b)). This fact is manifested by the isotropy of the strain-rate fluctuations mentioned in the context of Fig. 5b. In contrast, the distributions of the "parallel" and "perpendicular" components of the strain rate in the nonlinear case are much more heterogeneous and significantly different from each other (see parts (c) and (d)). In this connection, it should be recalled that in nonlinear composites the deformation rate can localize in bands, which may become progressively thinner as the nonlinearity increases (see for example, Moulinec and Suquet, 1998). Across such bands, the tangential component of the velocity field varies significantly, resulting in large shear strain rate. More precisely, if the vectors $\mathbf{t}$ and $\mathbf{n}$ denote, respectively, the directions tangential and normal to the band, then $\varepsilon_{t n}$ increases with decreasing band width, but not $\left(\varepsilon_{n n}-\varepsilon_{t t}\right) / 2$. Indeed, it can be seen in part (c) that the "parallel" component of the strain localizes in (white) bands running across the specimen, which are found to seek the (weaker) fibers, remaining at the same time as parallel as possible to the directions of maximum macroscopic shear $\left(0^{\circ}\right.$ and $\left.90^{\circ}\right)$. Thus, the macroscopic deformation rate is being accommodated mainly by large deformation rates along these bands, outside of which the deformation rate is relatively small. These localization bands are responsible for the significant increase of $\operatorname{SD}^{(r)}\left(\varepsilon_{\|}\right)$observed in Figs. 5b and 6a. On the other hand, it can be seen in part (d) that the "perpendicular" component of the strain rate is small wherever the bands observed in part (c) are oriented at $0^{\circ}$ and $90^{\circ}$. However, when the bands "bend" in order to accommodate the randomness of the distribution of fibers, this component is also found to be very large, being positive (white) or negative (black) depending on the local orientation of the band. Thus, the fact that all bands are not perfectly "aligned" with the directions of maximum macroscopic shear in this case explains the significant increase of $\operatorname{SD}^{(r)}\left(\varepsilon_{\perp}\right)$ observed in Figs. 5b and 6a. Nonetheless, the fluctuations of the "parallel" component of the strain rate are larger than those of the "perpendicular" component as a consequence of the preferred orientation of the bands mentioned above. (In fact, the ratio $\mathrm{SD}^{(r)}\left(\varepsilon_{\perp}\right) / \mathrm{SD}^{(r)}\left(\varepsilon_{\|}\right)$provides a measure of the tortuosity of the bands, for low enough values of $m$, which is strongly dependent on the concentration of fibers.) Thus, by allowing strain localization, nonlinearity not only increases significantly the strain-rate fluctuations, but also induces anisotropy on them, even though the phases and their spatial distribution are isotropic.

In the perfectly plastic limit, the localization bands can turn into shear bands, across which the tangential component of the velocity field is discontinuous (Suquet, 1981). In this connection, it is recalled that in the case of perfectly plastic porous composites with periodic microstructures, the exact solution corresponds to shear bands passing through the pores (Drucker, 1966), which is consistent with the localization bands observed in Fig. 7c. In addition, it is noted that in the presence of shear bands, the fluctuations of certain components of the strain rate may become unbounded. (In perfect plasticity, the strain-rate tensor $\varepsilon$ is a bounded measure on $\Omega$, and therefore, its integral is finite but its $L^{2}$ 
norm may become unbounded (Suquet, 1981).) As already mentioned, the FFT results for the standard deviations of both components of the strain rate shown in Figs. $6 \mathrm{~b}$ and $5 \mathrm{~b}$ are seen to increase at an increasing rate as $m$ decreases. This strongly suggests that indeed the strain-rate fluctuations should blow up in the perfectly plastic limit.

Fig. 8 provides the corresponding maps of the components of the stress field for the case of highly nonlinear $(m=0.1)$ phases. Part (a) shows the deviation in each phase of the "parallel" component about its phase average, that is, $\sigma_{12}(\mathbf{x})-\bar{\sigma}_{12}^{(1)}$ in the matrix and $\sigma_{12}(\mathbf{x})-\bar{\sigma}_{12}^{(2)}$ in the fibers, while part (b) shows the "perpendicular" component $\left(\sigma_{11}-\sigma_{22}\right) / 2$, which fluctuates about zero in both phases. In the linear case, the stress components are simply equal to the corresponding strain-rate component multiplied by the flow stress (constant within each phase), and so the maps of the stress are very similar to those shown in Figs. 7a and b, and are omitted here. The important point to make, however, is that, in the linear case, the distributions of both components of the stress also exhibit similar degrees of heterogeneity, and this is manifested by the isotropy of the stress fluctuations mentioned in the context of Fig. 4b. When the phases are nonlinear, on the other hand, the distribution of the "perpendicular" component is seen to be more heterogeneous than the that of the "parallel" component. In addition, the "parallel" component (part (a)) is seen to exhibit, in the matrix, a pattern similar to that of the strain rate observed in Fig. 7c, but much less contrasted, taking the largest values along the localization bands mentioned in the context of that figure. This is not surprising, since in the perfectly plastic limit a necessary (local) condition for the development of a shear band running at $0^{\circ}$ or $90^{\circ}$ is that $\sigma_{\|}=\sigma_{0}^{(r)}$. Note that while the strain rate is becoming unbounded along these bands, the stress is becoming bounded by the flow stress in each phase, as $m \rightarrow 0$. This is why the strain-rate fluctuations shown in Figs. 5b and 6a blow up as $m \rightarrow 0$, whereas the stress fluctuations shown in Figs. $4 \mathrm{~b}$ and 6 a remain finite.

Histograms. Fig. 9 provides plots for the probability density functions of the components of the local fields in the matrix. The clear and dark circles represent the FFT results corresponding to the specific configuration shown in Fig. 2 (and not to the
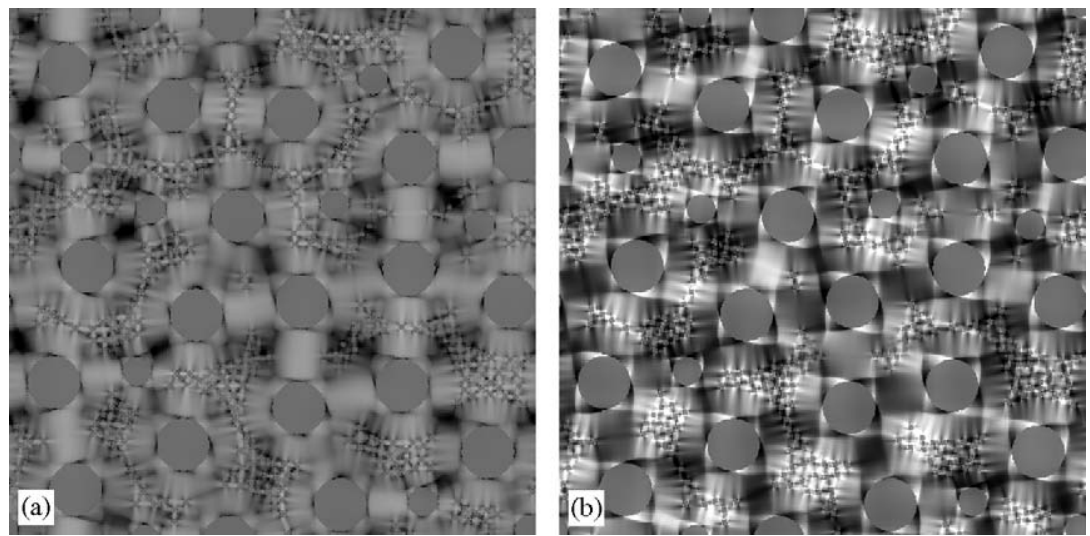

Fig. 8. Stress distribution in a power-law composite with weaker fibers $\left(\sigma_{0}^{(2)} / \sigma_{0}^{(1)}=0.2\right)$, subjected to in-plane shear $\bar{\sigma}_{12}$. The microstructure is the one shown in Fig. 2. Distribution for $m=0.1$ of the (a) "parallel" component $\sigma_{12}-\bar{\sigma}_{12}^{(r)}$, and (b) "perpendicular" component $\left(\sigma_{11}-\sigma_{22}\right) / 2$. The quantities are normalized by $\bar{\sigma}_{\mathrm{e}} / \sqrt{3}$. Black and white correspond, respectively, to values smaller than -1 and larger than 1 . 

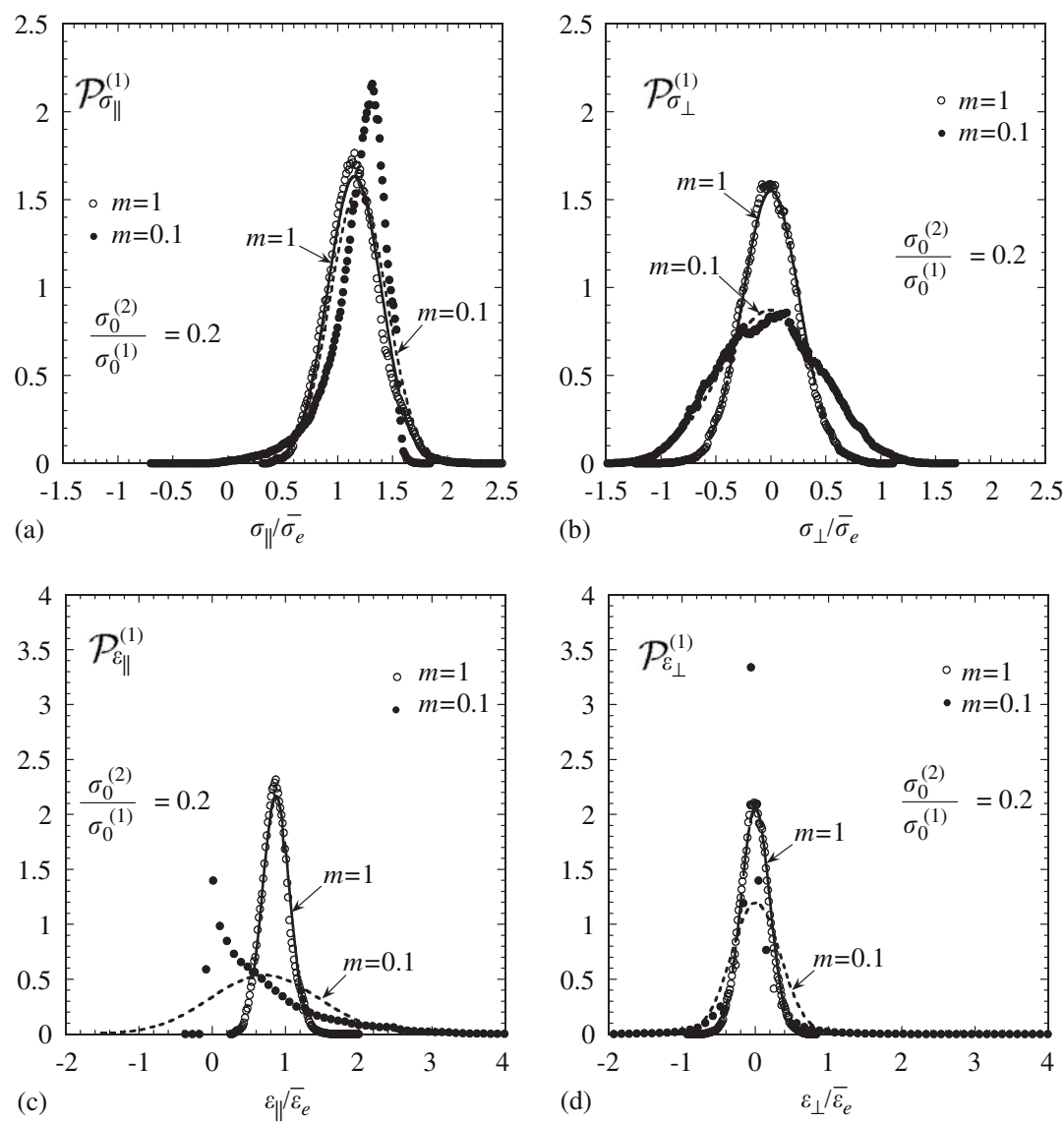

Fig. 9. FFT results for the probability density functions of the local fields for the case of weaker fibers, associated with the microstructure shown in Fig. 2. The clear and dark circles correspond, respectively, to exponents $m=1$ and $m=0.1$. The continuous and dashed lines represent, respectively, the Gaussian distributions whose mean and standard deviation are those obtained from the FFT simulations for $m=1$ and $m=0.1$. Distributions of (a) "parallel" $\left(\sigma_{\|} / \bar{\sigma}_{\mathrm{e}}\right)$ and (b) "perpendicular" $\left(\sigma_{\perp} / \bar{\sigma}_{\mathrm{e}}\right)$ components of the stress, (c) "parallel" ( $\left.\varepsilon_{\|} / \bar{\varepsilon}_{\mathrm{e}}\right)$ and (d) "perpendicular" $\left(\varepsilon_{\perp} / \bar{\varepsilon}_{\mathrm{e}}\right)$ components of the strain rate.

ensemble averages) with linear $(m=1)$ and highly nonlinear $(m=0.1)$ phases, respectively. In addition, the continuous and dashed lines represent, respectively, Gaussian distributions whose mean and standard deviations are those obtained from the FFT simulations for $m=1$ and $m=0.1$, which are included in order to verify the possible Gaussian character of the field distributions.

Parts (a) and (b) show, respectively, the distributions of the "parallel" and "perpendicular" components of the stress normalized by the equivalent macroscopic stress $\bar{\sigma}_{\mathrm{e}}$ (as given by expressions (34) with $z=\sigma_{\|} / \bar{\sigma}_{\mathrm{e}}$ and $z=\sigma_{\perp} / \bar{\sigma}_{\mathrm{e}}$ ). We begin by noting that in the linear case (clear circles), the distributions of both components of the stress are in very good agreement with the corresponding Gaussian distributions (continuous lines). (In this connection, it should be mentioned that while the Gaussian distributions vanish only at infinity, the distributions of the local fields vanish at finite values, which correspond 
to the maximum and minimum values that the fields take in the composite.) In addition, the distributions of both components are seen to be fairly similar to each other, except for a shift in abscissa, which is manifested by the isotropy of the stress fluctuations mentioned in the context of Fig. 4b. In the nonlinear case, on the other hand, while the distribution of the "perpendicular" component remains fairly Gaussian, the distribution of the "parallel" component is seen to become skewed to the right and to drop to zero rather abruptly for values of $\sigma_{\|} / \bar{\sigma}_{\mathrm{e}}$ larger than the mean. The latter is a manifestation of the fact that in the limiting case of perfect plasticity $(m \rightarrow 0)$, the stress components become bounded by the requirement $\sigma_{\mathrm{e}}^{2}=\sigma_{\|}^{2}+\sigma_{\perp}^{2} \leqslant\left(\sigma_{0}^{(r)}\right)^{2}$. Although this restriction applies to both stress components, the fact that only the distribution of the "parallel" component exhibits an abrupt drop follows from the fact that, unlike the "perpendicular" component, its mean value is greater than zero and therefore closer to the bound that develops in the limit $m \rightarrow 0$.

Parts (c) and (d) show, respectively, the corresponding distributions of the "parallel" and "perpendicular" components of the strain rate, normalized by the equivalent macroscopic strain rate $\bar{\varepsilon}_{\mathrm{e}}$ (as given by expressions (34) with $z=\varepsilon_{\|} / \bar{\varepsilon}_{\mathrm{e}}$ and $z=\varepsilon_{\perp} / \bar{\varepsilon}_{\mathrm{e}}$ ). In the linear case, the distributions of the strain-rate components are similar to those of the corresponding stress components, and therefore similar observations apply. Thus, the distributions of both strain-rate components (clear circles) are also found to be in good agreement with the corresponding Gaussian distributions (continuous lines). In the nonlinear case, on the other hand, the distributions of the "parallel" and "perpendicular" components (dark circles) are found to be very different from the corresponding Gaussian distributions (dashed lines). In particular, the distribution of the "parallel" component is seen to be highly skewed to the left with respect to its mean, being maximum at $\varepsilon_{\|} / \bar{\varepsilon}_{\mathrm{e}} \approx 0$ and exhibiting an abrupt drop for smaller values of $\varepsilon_{\|} / \bar{\varepsilon}_{\mathrm{e}}$ (see part (c)). In addition, this distribution is found to develop a tail in the range of large values of $\varepsilon_{\|} / \bar{\varepsilon}_{\mathrm{e}}$. In contrast, the distribution of the "perpendicular" component is found to remain symmetric about its mean, but it becomes more concentrated close to $\varepsilon_{\perp} / \bar{\varepsilon}_{\mathrm{e}}=0$ than in the linear case, and it develops tails in the range of large values of $\left|\varepsilon_{\perp} / \bar{\varepsilon}_{\mathrm{e}}\right|$. The tails developed by the strain-rate distributions correspond to the presence of very large strain rates in very small regions, i.e. localization bands, and the fact that these distributions are maximum at approximately 0 means that the deformation rate is relatively small for most regions (in the matrix) outside the bands. Thus, as the nonlinearity increases, the strain-rate distributions, especially that of the "parallel" component, progressively deviate further from a Gaussian distribution, due to the development of strain localization. It is worth noting that the reasons why the field distributions become non-Gaussian with nonlinearity are different for the strain rate than for the stress fields, and that the former is seen to be more sensitive in this regard than the latter.

\subsection{Fiber-reinforced composites}

Effective behavior. The various bounds and estimates for the effective flow stress $\widetilde{\sigma}_{0}$ of a fiber-reinforced composite are plotted in Fig. 3b, together with the FFT results as a function of the strain-rate sensitivity $m$, normalized by the flow stress of the matrix $\sigma_{0}^{(1)}$. The main observation in the context of this figure is that the SO estimates are found to be in good agreement with the FFT simulations, even for the smaller values of $m$. Thus, both methods yield a decreasing $\widetilde{\sigma}_{0}$ with decreasing values of $m$ (i.e., increasing nonlinearity), 
and in the perfectly plastic limit $(m \rightarrow 0)$ they predict no reinforcement effect due to the stronger fibers, i.e. $\widetilde{\sigma}_{0}=\sigma_{0}^{(1)}$. As pointed out by Drucker (1966), this is the correct limit if the arrangement of fibers allows for a shear plane passing through the matrix (see below for more details). Furthermore, this coincides with the Reuss lower bound, which is known to be optimal in this limit in $2 \mathrm{D}$ (see Garroni et al., 2001; Suquet, 2005). Also included in this figure are the "tangent second-order" (TSO) estimates and the "variational" (VAR) estimates. As already mentioned, the latter are rigorous upper bounds for all other nonlinear estimates of the HS type, and in particular for the SO and TSO estimates. Several comments are in order. First, the homogenization estimates all coincide for $m=1$ with the linear HS estimates, as they should, but give different predictions for other values of $m$. However, the SO and TSO estimates are found to be very similar for all values of $m$ in this case, and they coincide not only for $m=1$ but also for $m=0$. Thus the TSO estimates are also in good agreement with the FFT results in this case. The "variational" estimates, on the other hand, are found to overestimate the FFT results for all values of $m$ different than 1, and they even give a finite reinforcement effect in the limit $m \rightarrow 0$. It is further observed that, as anticipated, the TSO estimates exhibit a duality gap, but as opposed to what happens in the case of fiber-weakened composites, this gap is seen to be small for all values of $m$, and even vanishes in the perfectly plastic limit. In contrast, the SO estimates (11) and (13) are exactly equivalent for all values of $m$. Finally, it is noted that the scatter exhibited by the FFT results is found to be very small in this case as well.

Statistics of the local fields. Corresponding estimates for the phase averages and standard deviations of the stress field are shown in Fig. 10. Part (a) provides plots for the equivalent average stresses $\bar{\sigma}_{\mathrm{e}}{ }^{(r)}$ in each phase, normalized by the equivalent macroscopic stress $\bar{\sigma}_{\mathrm{e}}$. It can be seen in this figure that the FFT results show an average stress in the (stronger) fibers that is always higher than that in the matrix, as expected. However, the former is seen to decrease with increasing nonlinearity, while the latter is seen to increase, until they both coincide with $\bar{\sigma}_{\mathrm{e}}$ in the perfectly plastic limit. Among the homogenization estimates, the SO estimates are the most consistent ones with the FFT simulations, the agreement being good for all values of $m$. The $\operatorname{TSO}(U)$ estimates are found to give similar
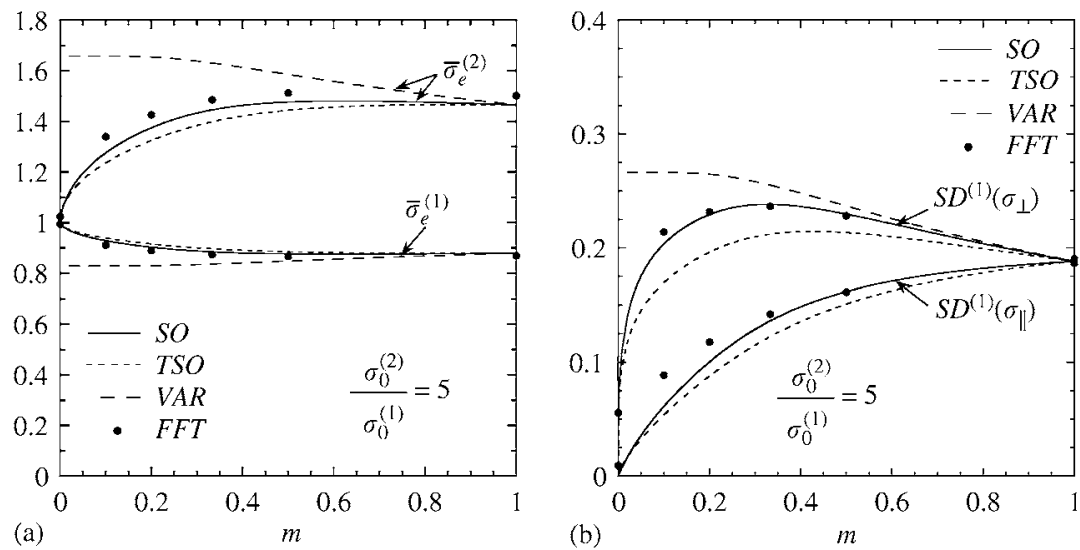

Fig. 10. Statistics of the stress field for the case of stronger fibers. (a) Equivalent average stresses in the matrix $\left(\bar{\sigma}_{\mathrm{e}}^{(1)}\right)$ and fibers $\left(\bar{\sigma}_{\mathrm{e}}^{(2)}\right)$. (b) Standard deviation (SD) of the "parallel" and "perpendicular" components of the stress field in the matrix. The results are normalized by $\bar{\sigma}_{\mathrm{e}}$. 
predictions, and even coincide with the SO estimates as $m \rightarrow 0$, as anticipated in the previous paragraph. In contrast, the trends exhibited by the "variational" estimates are seen to be inconsistent with the FFT results. Part (b) shows plots for the standard deviations of the "parallel" and "perpendicular" components of the stress in the matrix, normalized by the equivalent macroscopic stress $\bar{\sigma}_{\mathrm{e}}$. The main observation in the context of this figure is that, like in the previous subsection, the FFT simulations are found to give stress fluctuations that are isotropic in the linear case and become progressively more anisotropic as the nonlinearity increases, being larger for the "perpendicular" component than for the "parallel" component. However, unlike what happens in a fiber-weakened composite (cf. Fig. 4b), both the "parallel" and the "perpendicular" fluctuations are found to decrease for values of $m$ smaller than about 0.3 , almost vanishing in the perfectly plastic limit. It can be seen that the SO estimates are consistent with these observations, being in good agreement with the FFT results for all values of $m$. Note that in the limit $m \rightarrow 0$ these estimates predict vanishing fluctuations, which, together with the fact that $\bar{\sigma}_{\mathrm{e}}{ }^{(1)}=\bar{\sigma}_{\mathrm{e}}{ }^{(2)}=$ $\bar{\sigma}_{\mathrm{e}}$ (see part (a)), implies that the stress field tends to become uniform throughout the composite. It is further observed that the $\operatorname{TSO}(U)$ estimates exhibit the same trends as the FFT results, but they are not as close in general to them as the SO estimates. Finally, the "variational" estimates are seen to overestimate the stress fluctuations for all values of $m$ different than 1, and more importantly, they predict isotropic stress fluctuations for all values of $m$, thus being, once again, inconsistent with the FFT simulations.

Fig. 11 provides corresponding estimates for the phase averages and standard deviations of the strain-rate field. In part (a), plots are given for the equivalent average strain rate $\bar{\varepsilon}_{\mathrm{e}}{ }_{\mathrm{e}}^{(r)}$ in each phase, normalized by the equivalent macroscopic strain rate $\bar{\varepsilon}_{\mathrm{e}}$. It is observed in this figure that all homogenization estimates are in very good agreement with the FFT simulations, for all values of $m$. Thus, all methods are seen to predict an average strain rate in the (stronger) fibers which is lower than that in the matrix, and that decreases with decreasing $m$, until it vanishes in the limit $m \rightarrow 0$. Thus in this limit, the fibers behave like rigid inclusions. This is related to the well-known fact that nonlinearity enhances the
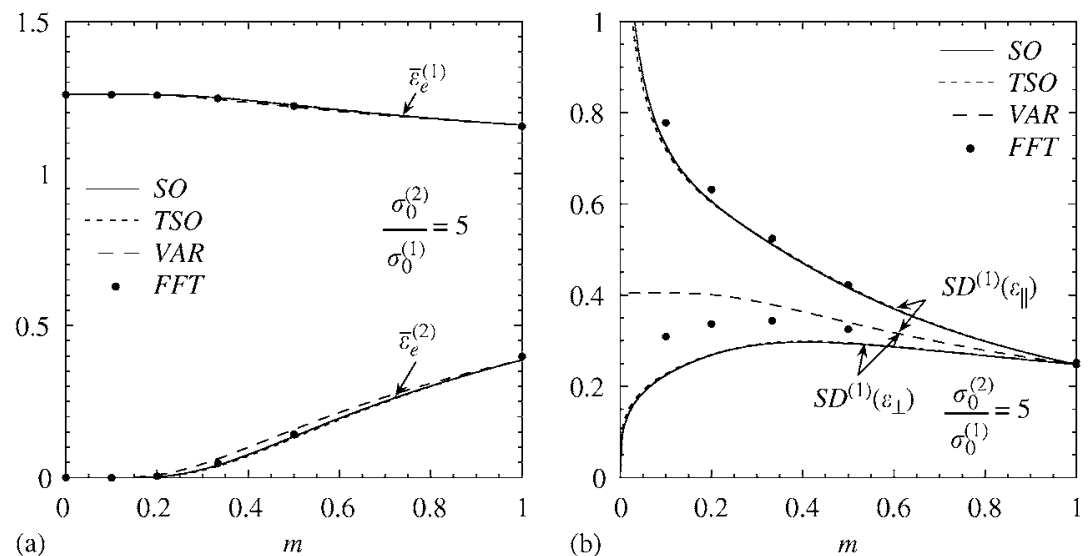

Fig. 11. Statistics of the strain-rate field for the case of stronger fibers. (a) Equivalent average strain rates in the matrix $\left(\bar{\varepsilon}_{\mathrm{e}}^{(1)}\right)$ and fibers $\left(\bar{\varepsilon}_{\mathrm{e}}^{(2)}\right)$. (b) Standard deviation (SD) of the "parallel" and "perpendicular" components of the strain-rate field in the matrix. The results are normalized by $\bar{\varepsilon}_{\mathrm{e}}$. 
contrast between the phases. In part (b), plots are given for the standard deviations of the strain-rate field in the matrix, normalized by the equivalent macroscopic strain rate $\bar{\varepsilon}_{\mathrm{e}}$. It is observed that, like in the case of fiber-weakened composites, the FFT simulations show strain-rate fluctuations which are isotropic in the linear case, and become progressively more anisotropic as the nonlinearity increases, being larger for the "parallel" component than for the "perpendicular" one. Moreover, the "parallel" fluctuations are seen to increase monotonically with nonlinearity here as well. However, unlike what happens in fiber-weakened composites, the "perpendicular" fluctuations are found to increase slightly in the range $0.3 \leqslant m \leqslant 1$, and decrease for smaller values of $m$. It can be seen that the SO estimates are consistent with these observations. Furthermore, it is seen that the SO estimates for the "parallel" strain-rate fluctuations are in good agreement with the FFT results for all values of $m$. On the other hand, the agreement may not be as good for the "perpendicular" strain-rate fluctuations, quantitatively, but the trends exhibited by both sets of results are seen to be fully consistent. It is also noted that, as $m \rightarrow 0$, the SO estimates predict infinite and vanishing strain-rate fluctuations in the "parallel" and "perpendicular" directions, respectively, which is consistent with strain localization along straight shear bands, as discussed below. It is further observed that the $\operatorname{TSO}(W)$ estimates are almost identical to the SO estimates for all values of $m$, so that the previous comments apply to these estimates as well. In contrast, the "variational" estimates are found to predict a slight increase of the strain-rate fluctuations with increasing nonlinearity, but more importantly, they predict, once again, isotropic strain-rate fluctuations for all values of $m$, which is inconsistent with the FFT simulations.

The corresponding standard deviations of the local fields in the fiber phase are shown in Fig. 6b. It can be seen that, unlike for the case of weaker fibers, the FFT simulations give stress fluctuations that are comparable to those in the matrix phase when $m=1$, and increase with increasing nonlinearity, becoming even larger than the stress fluctuations in the matrix phase for moderate nonlinearities. However, they decrease significantly as $m \rightarrow 0$, which together with the FFT results for the stress phase averages and fluctuations in the matrix (cf. Fig. 10) indicates that the stress field is quite uniform (and approximately equal to the macroscopic stress) in the perfectly plastic limit throughout the composite. On the other hand, the strain-rate fluctuations in the fiber phase are much smaller than those in the matrix phase (cf. Fig. 11b), for all values of $m$, and decrease with increasing nonlinearity. This is a consequence of the fact that, opposite to what happens in the case of weaker fibers, no strain localization occurs in the fibers when these are stronger than the matrix (see further below). The fact that the fluctuations of the local fields shown in Fig. 6b tend to be relatively small as $m \rightarrow 0$ may explain why the SO predictions based on the HS estimates for the LCC are in better agreement with the FFT results in this case than in the case of weaker fibers, where the strain-rate fluctuations in the fiber phase increase significantly for small values of $m$, and are therefore inconsistent with the HS hypothesis.

Distribution of local fields. Fig. 12 provides maps of the strain-rate field generated from the FFT simulations, for a composite with the microstructure shown in Fig. 2, for the case of highly nonlinear $(m=0.1)$ phases, subjected to in-plane shear (30). Part (a) shows the deviation in each phase of the "parallel" component about its phase average, that is, $\varepsilon_{12}(\mathbf{x})-\bar{\varepsilon}_{12}{ }^{(1)}$ in the matrix and $\varepsilon_{12}(\mathbf{x})-\bar{\varepsilon}_{12}{ }^{(2)}$ in the fibers, while part (b) shows the "perpendicular" component $\left(\varepsilon_{11}-\varepsilon_{22}\right) / 2$, which fluctuates about zero in both phases. The corresponding maps for the linear case $(m=1)$ are, for the purposes of the comparisons of interest here, qualitatively similar to those shown in Figs. 7a and b for fiber-weakened 

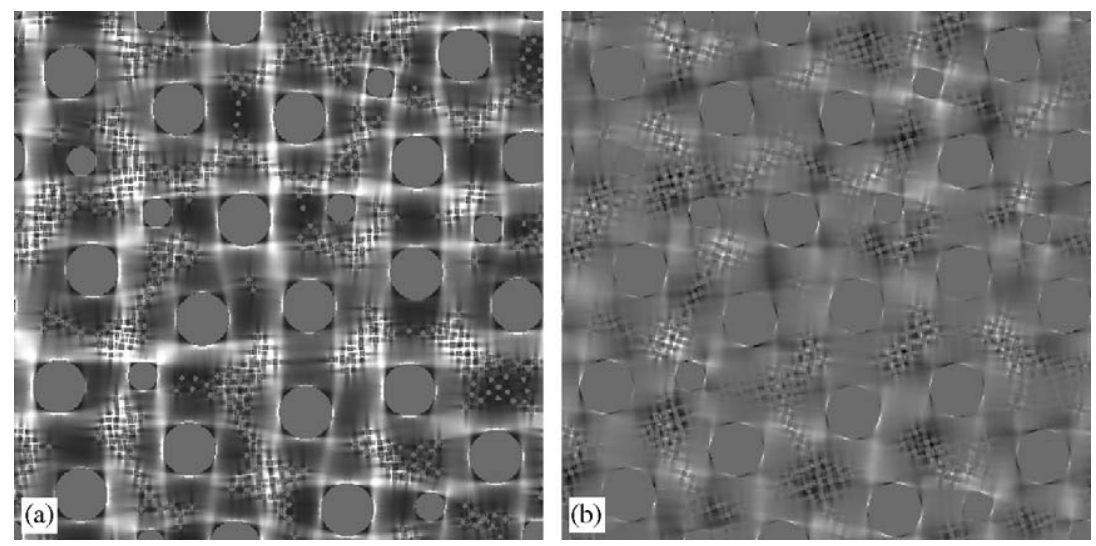

Fig. 12. Strain-rate distribution in a power-law composite with stronger fibers $\left(\sigma_{0}^{(2)} / \sigma_{0}^{(1)}=5\right)$, subjected to inplane shear $\bar{\sigma}_{12}$. The microstructure is the one shown in Fig. 2, and the exponent is $m=0.1$. Distribution of the (a) "parallel" component $\varepsilon_{12}-\bar{\varepsilon}_{12}^{(r)}$, and (b) "perpendicular" component $\left(\varepsilon_{11}-\varepsilon_{22}\right) / 2$. The quantities are normalized by $(\sqrt{3} / 2) \bar{\varepsilon}_{\mathrm{e}}$. Black and white correspond, respectively, to values smaller than -2 and larger than 2 .

composites, and are therefore omitted for brevity. Several comments are relevant in the context of this figure. First, as it has already been observed in the case of weaker fibers, while in the linear case the distributions of both components of the strain rate are rather diffuse and exhibit similar degrees of heterogeneity, in the nonlinear case the distributions of the "parallel" and "perpendicular" components are much more heterogeneous and significantly different from each other. As already explained in the previous subsection, this is due to the fact that for nonlinear materials the deformation rate tends to localize in thin bands running across the specimen. However, unlike what happens for the case of weaker fibers, these localization bands are found here to avoid the (stronger) fibers, remaining at the same time as parallel as possible to the directions of maximum macroscopic shear $\left(0^{\circ}\right.$ and $\left.90^{\circ}\right)$. This is the reason why in this case the strain-rate fluctuations, shown in Figs. $11 \mathrm{~b}$ and $6 \mathrm{~b}$, while increasing in the matrix with increasing nonlinearity, actually decrease in the fibers. In addition, it is observed that, as for the case of weaker fibers, the bands tend to "bend" in order to accommodate the randomness of the distribution of fibers, but they remain straighter than when they seek the fibers (at least at this concentration of fibers). This fact helps explain why the "perpendicular" component shown in Fig. 12b is not as localized as that shown in Fig. 7d. In fact, the trend exhibited by the FFT results for $\operatorname{SD}^{(1)}\left(\varepsilon_{\perp}\right)$ shown in Fig. 11b suggests that the fluctuations of the "perpendicular" component of the strain rate in the matrix actually decrease for smaller values of $m$, unlike in the case of weaker fibers (cf. Fig. 6b).

In order to put these results in context, it is useful to recall the following result for ideally plastic materials reinforced by stronger fibers (Drucker, 1966; Suquet, 1993): if the arrangement of the fibers in the composite is such that it is possible to pass (straight) planes through the matrix that are aligned with the shear loading, then the exact result corresponds to straight shear bands along these planes. In this case, the effective flow stress $\widetilde{\sigma}_{0}$ would be exactly that of the matrix, as it has already been mentioned. In addition, the corresponding strain-rate fluctuations in the matrix would be such that $\operatorname{SD}^{(1)}\left(\varepsilon_{\|}\right) \rightarrow \infty$ and $\mathrm{SD}^{(1)}\left(\varepsilon_{\perp}\right) \rightarrow 0$, while the strain rate in the fibers would be exactly zero. Also, the stress field 
would be uniform throughout the composite. Now, it is observed that the trends seen for the "second-order" HS predictions in the limit as $m \rightarrow 0$ are entirely consistent with this result (regardless of the concentration of fibers). On the other hand, for the specific random microstructures considered in the FFT simulations, the probability of finding such (straight) shear planes is expected to be small at relatively small fiber concentrations, and to vanish at sufficiently high fiber concentrations. Indeed, at the intermediate volume fraction considered here $(0.20626)$, many realizations of the composite do not allow for such straight shear planes, and therefore the above-mentioned results cannot be expected to apply. This is the reason why the FFT results do not agree precisely with the "secondorder" HS estimates in the ideally plastic limit. However, the fact that, at this volume fraction, nearly straight bands can develop explains why the FFT results are still in fairly good agreement with the "second-order" HS estimates in this case.

Fig. 13 provides the corresponding maps of the stress field for the case of highly nonlinear $(m=0.1)$ phases. Part (a) shows the deviation of the "parallel" component in each phase about its phase average, that is, $\sigma_{12}(\mathbf{x})-\bar{\sigma}_{12}^{(1)}$ in the matrix and $\sigma_{12}(\mathbf{x})-\bar{\sigma}_{12}^{(2)}$ in the fibers, while part (b) shows the "perpendicular" component $\left(\sigma_{11}-\sigma_{22}\right) / 2$, which fluctuates about zero in both phases. It is recalled that in the linear case, the maps of the stress are qualitatively similar to those of the strain shown in Figs. 7a and b, and are omitted here. The important point to make, though, is that, when the phases are linear, the distribution of both components of the stress also exhibit similar degrees of heterogeneity, and this is manifested by the isotropy of the stress fluctuations shown in Fig. 10b. On the other hand, when the phases are nonlinear, the distributions of the "parallel" and "perpendicular" components of the stress are found to be very different from each other. In particular, the "parallel" component shown in part (a) is seen to be quite homogeneous throughout the specimen. In fact, as $m \rightarrow 0$ it becomes progressively more homogeneous, tending to the flow stress of the matrix everywhere. Note that this level of stress is large enough to produce localization bands in the matrix, but does not produce any deformation in the (stronger) fibers (cf. Figs. 12c and d). In contrast, the distribution of the
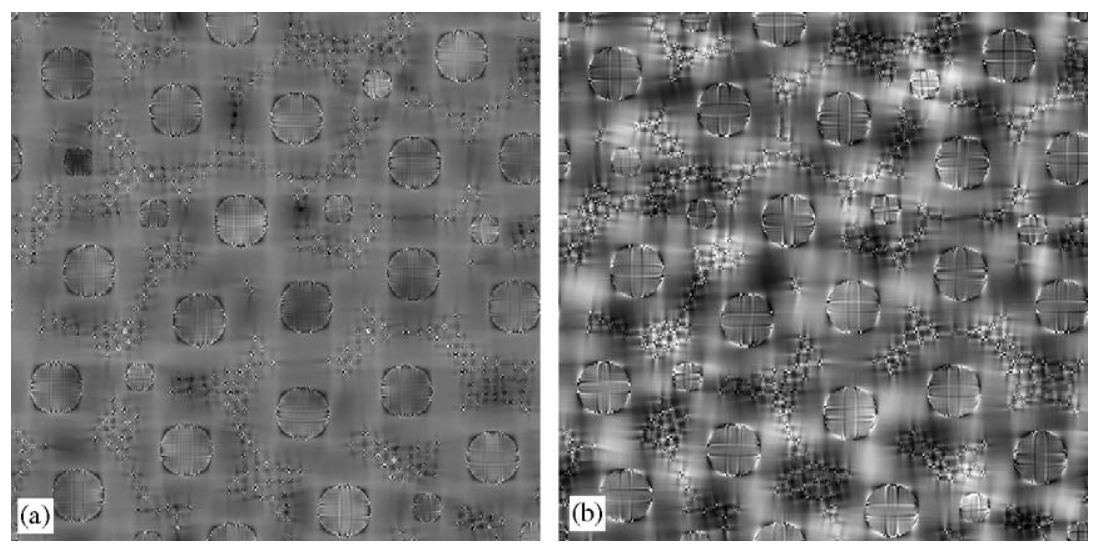

Fig. 13. Stress distribution in a power-law composite with stronger fibers $\left(\sigma_{0}^{(2)} / \sigma_{0}^{(1)}=5\right)$, subjected to in-plane shear $\bar{\sigma}_{12}$. The microstructure is the one shown in Fig. 2, and the exponent is $m=0.1$. Distribution of the (a) "parallel" component $\sigma_{12}-\bar{\sigma}_{12}^{(r)}$, and (b) "perpendicular" component $\left(\sigma_{11}-\sigma_{22}\right) / 2$. The quantities are normalized by $\bar{\sigma}_{\mathrm{e}} / \sqrt{3}$. Black and white correspond, respectively, to values smaller than -0.68 and larger than 0.68 . 
"perpendicular" component exhibits a much more heterogeneous and complicated pattern, especially in the matrix. We do not have an explanation for such a pattern. In any event, the differences between these distributions is what gives rise to the anisotropy of the stress fluctuations mentioned in the context of Fig. $10 \mathrm{~b}$ (for $m=0.1$ ). Finally, it is also noted that both components of the stress are seen to exhibit a "cross" pattern inside the fibers, but this could be an artifact of the numerical simulations related to the "pixelation" of the fiber boundaries.

Histograms. Fig. 14 provides plots for the probability density functions of the components of the local fields in the matrix. The clear and dark circles represent the FFT results corresponding to the specific configuration shown in Fig. 2 (and not ensemble averages) with linear $(m=1)$ and highly nonlinear $(m=0.1)$ phases, respectively. In addition, the continuous and dashed lines represent, respectively, Gaussian distributions
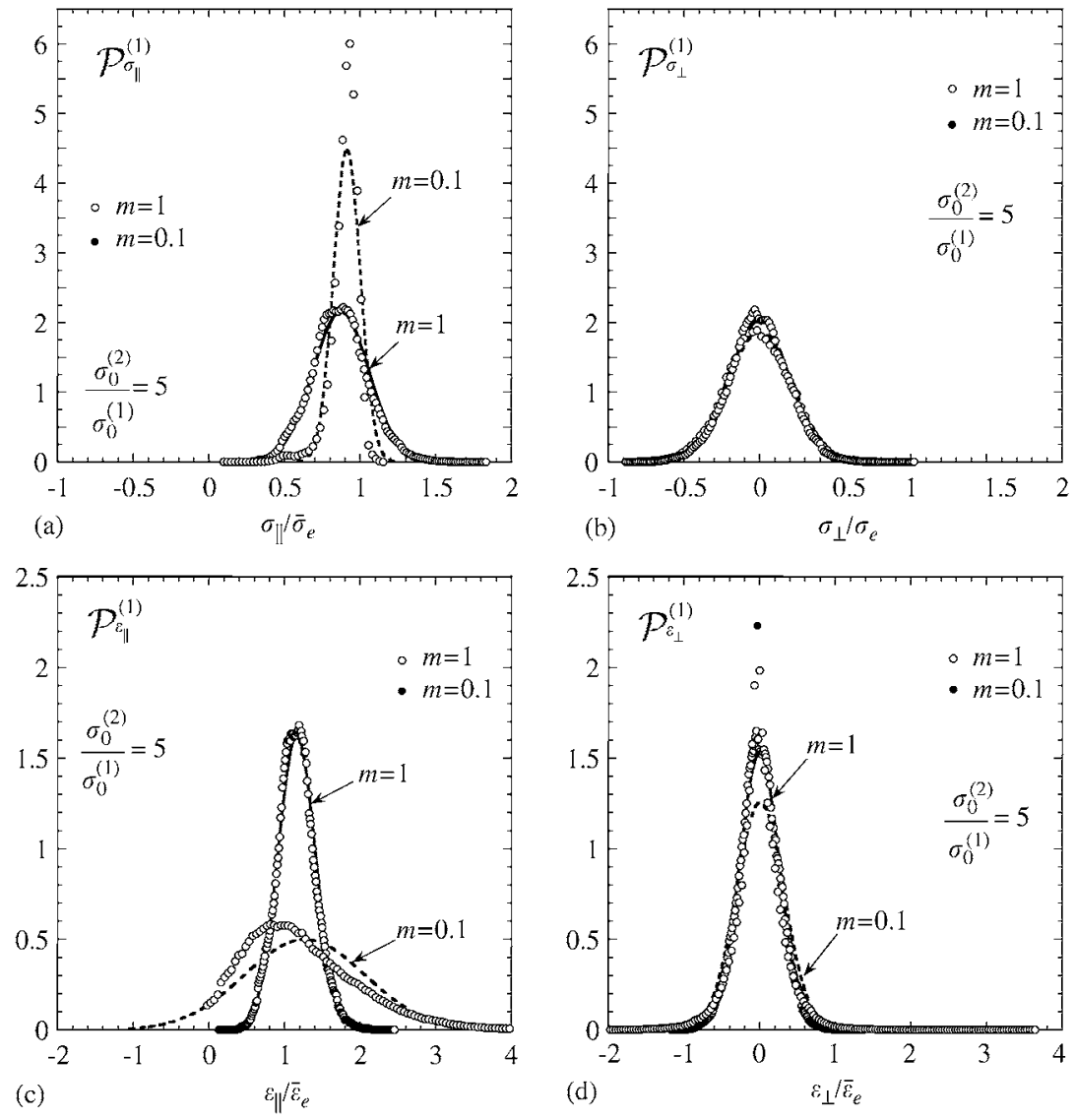

Fig. 14. FFT results for the probability density functions of the local fields for the case of stronger fibers, associated with the microstructure shown in Fig. 2. The clear and dark circles correspond, respectively, to exponents $m=1$ and $m=0.1$. The continuous and dashed lines represent, respectively, the Gaussian distributions whose mean and standard deviation are those obtained from the FFT simulations for $m=1$ and $m=0.1$. Distributions of (a) "parallel" $\left(\sigma_{\|} / \bar{\sigma}_{\mathrm{e}}\right)$ and (b) "perpendicular" $\left(\sigma_{\perp} / \bar{\sigma}_{\mathrm{e}}\right)$ components of the stress, (c) "parallel" $\left(\varepsilon_{\|} / \bar{\varepsilon}_{\mathrm{e}}\right)$ and $(\mathrm{d})$ "perpendicular" $\left(\varepsilon_{\perp} / \bar{\varepsilon}_{\mathrm{e}}\right)$ components of the strain rate. 
whose mean and standard deviation are those obtained from the FFT simulations for $m=1$ and $m=0.1$.

Parts (a) and (b) show, respectively, the distributions of the "parallel" and "perpendicular" components of the stress normalized by the equivalent macroscopic stress $\bar{\sigma}_{\mathrm{e}}$. As in the case of weaker fibers, when the phases are linear, the distributions of both components of the stress (clear circles) are found to be in very good agreement with the corresponding Gaussian distributions (continuous lines). In addition, both distributions are seen to be very similar to each other, which is manifested by the isotropy of the stress fluctuations mentioned in the context of Fig. 10b. When the phases are nonlinear, on the other hand, the distributions of both components of the stress (dark circles) are seen to be very different from each other. Thus, it is observed that, as for the case of weaker fibers, the distribution of the "parallel" component of the stress is in disagreement with the corresponding Gaussian distribution (see part (a)), while the "perpendicular" component remains in good agreement with the corresponding Gaussian distribution (see part (b)). As already mentioned in the context of Fig. 9, this is due to the fact that in the limiting case of perfect plasticity $(m \rightarrow 0)$, the stress becomes bounded by the requirement $\sigma_{\mathrm{e}}^{2} \leqslant\left(\sigma_{0}^{(r)}\right)^{2}$.

Parts (c) and (d) show, respectively, the corresponding distributions of the "parallel" and "perpendicular" components of the strain rate, normalized by the equivalent macroscopic strain rate $\bar{\varepsilon}_{\mathrm{e}}$. It can be seen that while in the linear case the distributions of the "parallel" and "perpendicular" components of the strain rate are seen to be fairly Gaussian, in the nonlinear case they are found to be very different from the corresponding Gaussian distributions. More specifically, the distribution of the "parallel" component is seen to become skewed to the left with respect to its mean, and to develop a tail for large values of $\varepsilon_{\|} / \bar{\varepsilon}_{\mathrm{e}}$ (see part (c)). In contrast, the "perpendicular" component is seen to remain fairly symmetric with respect to its mean, becoming more concentrated at $\varepsilon_{\perp} / \bar{\varepsilon}_{\mathrm{e}}=0$ than in the linear case, and to develop tails in the range of large values of $\left|\varepsilon_{\perp} / \bar{\varepsilon}_{\mathrm{e}}\right|$ (see part (d)). As already pointed out in the context of weaker fibers, these tails are due to the presence of strain localization bands, since the bands represent thin regions where the strain rate takes very large values. However, unlike what is observed in the case of weaker fibers, the distribution of the "parallel" component exhibits a maximum at $\varepsilon_{\|} / \bar{\varepsilon}_{\mathrm{e}} \approx 1$, as opposed to $\approx 0$ (cf. Fig. 9c). This means that, while the strain rate in most regions outside the localization bands is relatively small when the fibers are weaker than the matrix, it is approximately equal to the macroscopic strain rate when the fibers are stronger than the matrix, at least at these values of the strain-rate sensitivity and fiber concentration. This may be related to the fact that the localization bands tend to be more uniformly distributed in the matrix phase for the case of stronger fibers than for the case of weaker fibers, where the bands choose fewer paths through the matrix (see Figs. 7c and 12c). Finally, it is noted that, like in the case of weaker fibers, the strain-rate distributions are seen to be much more sensitive to nonlinearity than the stress distributions, in the sense that they deviate more significantly from the corresponding Gaussian distributions.

\section{Conclusions}

This paper has presented a combined numerical-theoretical study of the macroscopic behavior and local field distributions for a special class of nonlinear composite materials with random "particulate" microstructures. The numerical simulations were carried out using the FFT method developed by Moulinec and Suquet (1994, 1998), and have the 
advantage of leading to (numerically) exact results, as well as to complete information on the field distributions, at least at the level of one realization of the microstructure. Information on the statistics of the fields were obtained by performing ensemble averages over several different realizations. The theoretical results were obtained by means of the "second-order" homogenization method, proposed by Ponte Castañeda (2002a), making use of suitably chosen estimates for the effective behavior of a linear comparison composite (LCC) whose properties are determined by the method itself. The main advantage of the method is that the resulting estimates are analytical, up to some nonlinear algebraic equations, which can be solved numerically with negligible computational effort. On the other hand, the theoretical predictions introduce approximations both at the level of the estimate for the effective behavior of the LCC, as well as at the level of the linearization itself. The main findings of this work are as follows.

The fluctuations of the strain-rate and stress fields, as measured by the standard deviations of these quantities, were found to generally increase and to become anisotropic, with increasing nonlinearity (decreasing values of strain-rate sensitivity $m$ ), in agreement with earlier theoretical predictions using the "second-order" method (Ponte Castañeda, 2002b; Idiart and Ponte Castañeda, 2003). More specifically, for the case of softer particles, the FFT simulations show that both the "parallel" and "perpendicular" components of the fluctuations of the strain-rate field tend to increase, with the parallel component increasing faster than the perpendicular component, and tending to become unbounded in the ideally plastic limit $(m=0)$. These results were found to be consistent with the localization of the deformation pattern in the strongly nonlinear composite, where the localization bands "bend" seeking out minimum dissipation paths through the softer inclusions (see Fig. 7). In this case, the fluctuations of the corresponding stress field were also found to increase with increasing nonlinearity, with the difference that this time the perpendicular components of the fluctuations were found to be larger, and that the fluctuations remain bounded in the ideally plastic limit. For the harder-particle case, similar trends were observed for the strain-rate and stress fluctuations, but this time the perpendicular component of the strain-rate, as well as of both components of the stress, tends to become smaller for sufficiently low values of the strain-rate sensitivity parameter. These observations were thought to be related to the fact that, contrary to the softerparticle case, in the harder-particle case, the localized bands tend to avoid the particles and develop in the gaps between the inclusions (see Fig. 12). For the relatively low concentration of fibers considered in this study (approximately 20\%), the macroscopic deformation can be accommodated by nearly straight bands, requiring large fluctuations in the parallel component of the strain rate, but relatively smaller fluctuations in the perpendicular component of the strain rate, as well as for both components of the stress.

Even though the "second-order" method, being only a homogenization theory, is not able to capture detailed information about the distribution of the local fields in the composites, it was found that the method gives remarkably accurate predictions not only for the averages of the stress and strain-rate fields in the phases, but also for the standard deviations of the fields in the composite, including the above-mentioned strong dependence of the anisotropy of the fields on the nonlinearity of the material. Even though the "second-order" method could never mimic the highly localized patterns of deformation shown in Figs. 7 and 12, somehow it is able to capture the signature of these localized fields through fairly accurate predictions for the standard deviations of the fields. Thus, the "second-order" predictions are consistent with the fact that the parallel component of the 
strain rate localizes more than the corresponding perpendicular component, as well as with the opposite prediction that the perpendicular component of the stress fluctuates more than the corresponding parallel component. The theoretical predictions even reflect the more subtle relative differences between the deformation and stress patterns for the softerand harder-particle cases.

As already anticipated in an earlier publication (Moulinec and Suquet, 2003), the FFT results (see Figs. 9 and 14) show that the probability distributions of the fields (in the matrix phase) become progressively distorted away from Gaussian with increasing nonlinearity. This suggests that the "second-order" homogenization theory using a linear comparison composite - and therefore incorporating only information on the first and second moments of the fields - would not be able to capture the higher moments required to explain the strongly non-Gaussian behavior for the higher nonlinearities. However, the theory somehow does the best it can with the information that it has at its disposal, and it would seem that the higher moments which would be required to capture accurately the non-Gaussian distribution of the fields are perhaps not essential to obtain fairly accurate estimates for the first two moments of the fields (except perhaps in the ideally plastic limit). An additional observation in the context of the probability distributions is that while the distributions of some of the components of the fields become strongly non-Gaussian with increasing nonlinearity, as expected, other components actually remain fairly Gaussian. Thus, the parallel component of the stress becomes skewed to the right, which can be explained by the fact that the stress develops a bound with increasing nonlinearity, while the parallel component of the strain-rate becomes skewed to the left, which can be explained by the fact that the strain-rate field localizes and hence develops a long tail for large values of the strain rate. On the other hand, the perpendicular component of the stress is found to remain fairly Gaussian even for a relatively low value of $m(0.1)$, while the perpendicular component of the strain rate is fairly symmetric, and although strictly not Gaussian, a Gaussian would not be a bad approximation.

As already mentioned, the "second-order" method appears to give fairly accurate estimates for the first and second moments of the stress and strain-rate fields. In addition, the "second-order" estimates for the macroscopic behavior also appear to be quite good. Of course, the agreement is not perfect, especially for the stronger nonlinearities, and it is generally better for the harder-particle case than for the softer-particle case, which appears to be more sensitive. The worse agreement for the softer-particle case than for the harderparticle case is probably related to the fact that the field fluctuations generated by the FFT simulations in the inclusion phase are much larger (in relative terms) for the softer-particle case. This fact is inconsistent with the assumption that the fluctuations are small (in fact, vanish) implicit in the use of the HS estimates to estimate the effective behavior of the LCC (for use with the "second-order" method). Indeed, the fact that the deformation fields localize in bands that tend to go through the inclusion phase in the softer-particle case implies that the fields are forced to fluctuate significantly in the inclusion phase. Therefore, in retrospect, the use of the HS approximation for the LCC in the softer-particle case is probably not fully justified, at least for strong nonlinearities. In this connection, it is important to emphasize that the choice of the HS approximation is strictly appropriate in the context of the composite cylinder assemblage (CCA) microstructure only for linear systems with isotropic phases. In fact, the LCC used to estimate the effective behavior of the nonlinear composite has anisotropic phases, and so the use of the HS approximation is not necessarily justified a priori. On the other hand, the fact that the "second-order" 
estimates for the harder-particle case, where the HS estimates for the LCC are more appropriate (because the fluctuations in the fibers are smaller in this case), suggest that the "second-order" method itself has the capability of giving good estimates for the effective behavior and field fluctuations in the nonlinear composite, provided that sufficiently accurate estimates are available for the LCC. This suggests that improved "second-order" estimates could be generated for the softer-particle case if use is made of more appropriate estimates for the effective behavior of the LCC. One possibility, which will be left for future work, is to estimate numerically the exact effective behavior of the LCC, and to generate improved estimates for the effective behavior of the nonlinear composite still using the "second-order" method. This type of comparison is certainly feasible, and has already been attempted in earlier work (Moulinec and Suquet, 2004) in connection with the variational approximation.

Now, if the differences observed between the "second-order" predictions and the FFT simulations at the larger nonlinearities could be largely attributed to inaccuracies associated with the computation of the effective behavior of the LCC, this would suggest that the "second-order" method has the capability to give fairly accurate predictions not only for the first and second moments of the local fields, but also for the macroscopic behavior. Since, in principle, the macroscopic behavior of a nonlinear composite would be expected to also depend on the higher moments of the fields, it would follow that the effective behavior of such nonlinear composite systems is controlled primarily by the first two moments of the fields (which is the only information available to the "second-order" method making use of an LCC). If this observation were confirmed by more careful comparisons between the "second-order" estimates and full-field simulations, it could help explain why the "second-order" estimates for the macroscopic behavior turn out to be so good, at least for this class of microstructures.

Concerning comparisons of the "second-order" estimates with earlier types of estimates, it has been found that the "second-order" estimates are the most accurate in an overall sense. In particular, the variational method (Ponte Castañeda, 1991), or modified secant method (Suquet, 1995), leads to qualitatively incorrect predictions for the phase averages and standard deviations of the field fluctuations in the phases. Thus, for example, it gives rather inaccurate predictions for the phase averages of the strain-rate field in the softerparticle case, while it gives inaccurate predictions for the phase averages of the stress field in the harder-particle case. It also misses out the strong dependence of the field fluctuations on nonlinearity, including the pronounced anisotropy of the fluctuations, which can be attributed to the use of an LCC with local isotropic behavior (in the variational method). The variational predictions for the macroscopic behavior are also less accurate than the corresponding "second-order" predictions, although they have the redeeming feature that they provide bounds, while the "second-order" predictions do not. While the earlier "tangent second-order" estimates (Ponte Castañeda, 1996) are only slightly less accurate than the more recent "second-order" estimates (Ponte Castañeda, 2002a) for the harderparticle case, the "tangent second-order" estimates are clearly less accurate for the softerparticle case. Thus, while the "tangent second-order" estimates give very good predictions for weak nonlinearities, they become progressively less accurate, and can also give qualitatively incorrect predictions for large values of the nonlinearity. In addition, the "tangent" predictions for the effective behavior exhibit a large duality gap in the ideally plastic limit, which can be associated with the large fluctuations that develop in the fields in this case. It should also be emphasized that the "second-order" estimates derived in this 
work make use of a different choice for the "reference tensor" than the one proposed originally by Ponte Castañeda (2002a), and used by Idiart and Ponte Castañeda (2003). This new choice for the "reference tensor" appears to give improved results, in the sense that it gives better overall agreement with the numerical results, but the optimal choice of this variable remains an open problem.

Finally, it should be mentioned that similar comparisons between full-field simulations and homogenization estimates for the macroscopic behavior and field fluctuations in a special class of two-dimensional viscoplastic polycrystals have been carried out recently by Lebensohn et al. (2004) (see also Bhattacharya and Suquet, 2005). In that work, use was made of the standard self-consistent approximation for the LCC, which is better suited to "granular" microstructures. However, the conclusions of that work are entirely consistent with the conclusions of the present work, in that it was also found in the polycrystalline work that the "second-order" estimates improved - often in qualitative terms - on earlier types of homogenization estimates, and that these estimates are rather accurate when compared to full-field numerical simulations. Given the fact that the "second-order" method appears to give accurate estimates for these two rather different but also special types of composites, it is conjectured that the method may also lead to accurate results for even more general types of composites.

\section{Acknowledgements}

This research was carried out within the context of an NSF-CNRS international collaboration (NSF Grant no. OISE-0231867). The work of M.I.I. and P.P.C. was supported by NSF Grant CMS-02-01454.

\section{References}

Bhattacharya, K., Suquet, P., 2005. A model problem concerning recoverable strains in shape memory polycrystals. Proc. R. Soc. London A 461, 2797-2816.

Bobeth, M., Diener, G., 1987. Static elastic and thermoelastic field fluctuations in multiphase composites. J. Mech. Phys. Solids 35, 37-149.

Bornert, M., Hervé, E., Stolz, C., Zaoui, A., 1994. Self-consistent approaches and strain heterogeneities in twophase elastoplastic materials. App. Mech. Rev. 47 (1), 66-76.

Cheng, H., Torquato, S., 1997. Electric-field fluctuations in random dielectric composites. Phys. Rev. B 56, 8060-8068.

Drucker, D.C., 1966. The continuum theory of plasticity on the macroscale and the microscale. J. Mater. 1, $873-910$.

Garroni, A., Nesi, V., Ponsiglione, M., 2001. Dielectric breakdown: optimal bounds. Proc. R. Soc. London A 457, 2317-2335.

Hashin, Z., Rosen, B.W., 1964. The elastic moduli of fiber-reinforced materials. J. App. Mech. 31, Trans. ASME E 86, 223-232.

Hashin, Z., Shtrikman, S., 1963. A variational approach to the theory of the elastic behavior of multiphase materials. J. Mech. Phys. Solids 11, 127-140.

Hu, G., 1996. A method of plasticity for general aligned spheroidal void of fiber-reinforced composites. Int. J. Plasticity 12, 439-449.

Idiart, M., Ponte Castañeda, P., 2003. Field fluctuations and macroscopic properties for nonlinear composites. Int. J. Sol. Struct. 40, 7015-7033.

Idiart, M., Ponte Castañeda, P., 2005. Second-order estimates for nonlinear isotropic composites with spherical pores and rigid particles. C. R. Mec. 333, 147-154.

Kanit, T., Forest, S., Galliet, I., Mounoury, V., Jeulin, D., 2003. Determination of the size of the representative volume element for random composites: statistical and numerical approach. Int. J. Sol. Struct. 40, 3647-3679. 
Lebensohn, R.A., Liu, Y., Ponte Castañeda, P., 2004. Macroscopic properties and field fluctuations in model power-law polycrystals: full-field solutions versus self-consistent estimates. Proc. R. Soc. London A 460, 1381-1405.

Michel, J., Moulinec, H., Suquet, P., 2001. A computational scheme for linear and non-linear composites with arbitrarily phase contrast. Int. J. Numer. Methods Eng. 52, 139-160.

Moulinec, H., Suquet, P., 1994. A fast numerical method for computing the linear and nonlinear properties of composites. C. R. Acad. Sci. Paris II 318, 1417-1423.

Moulinec, H., Suquet, P., 1998. A numerical method for computing the overall response of nonlinear composites with complex microstructure. Comput. Methods Appl. Mech. Eng. 157, 69-94.

Moulinec, H., Suquet, P., 2003. Intraphase strain heterogeneity in nonlinear composites: a computational approach. Eur. J. Mech. A 22, 751-770.

Moulinec, H., Suquet, P., 2004. Homogenization for nonlinear composites in the light of numerical simulations. In: Ponte Castañeda, P., et al. (Eds.), Nonlinear Homogenization and Its Applications to Composites, Polycrystals and Smart Materials. Kluwer Academic Publishers, The Netherlands, pp. 193-223.

Parton, V.Z., Buryachenko, V.A., 1990. Stress fluctuations in elastic composites. Sov. Phys. Dokl. 35, $191-193$.

Pellegrini, Y.P., 2000. Field distributions and effective-medium approximation for weakly nonlinear media. Phys. Rev. B 61, 9365-9372.

Pellegrini, Y.P., 2001. Self-consistent effective-medium approximation for strongly nonlinear media. Phys. Rev. B 64, 134211.

Ponte Castañeda, P., 1991. The effective mechanical properties of nonlinear isotropic composites. J. Mech. Phys. Solids 39, 45-71.

Ponte Castañeda, P., 1996. Exact second-order estimates for the effective mechanical properties of nonlinear composite materials. J. Mech. Phys. Solids 44, 827-862.

Ponte Castañeda, P., 2002a. Second-order homogenization estimates for nonlinear composites incorporating field fluctuations: I-Theory. J. Mech. Phys. Solids 50, 737-757.

Ponte Castañeda, P., 2002b. Second-order homogenization estimates for nonlinear composites incorporating field fluctuations: II-Applications. J. Mech. Phys. Solids 50, 759-782.

Ponte Castañeda, P., Suquet, P., 1998. Nonlinear composites. Adv. Appl. Mech. 34, 171-302.

Suquet, P., 1981. Sur les équations de la plasticité: existence et régularité des solutions. J. de Méc. 20 , 3-39.

Suquet, P., 1993. Overall potentials and extremal surfaces of power law or ideally plastic materials. J. Mech. Phys. Solids 41, 981-1002.

Suquet, P., 1995. Overall properties of nonlinear composites: a modified secant moduli theory and its link with Ponte Castañeda's nonlinear variational procedure. C. R. Acad. Sci. Paris II 320, 563-571.

Suquet, P., 2005. On the effect of small fluctuations in the volume fraction of constituents on the effective properties of composites. C. R. Mec. 333, 219-226.

Suquet, P., Ponte Castañeda, P., 1993. Small-contrast perturbation expansions for the effective properties of nonlinear composites. C. R. Acad. Sci. Paris II 317, 1515-1522.

Talbot, D.R.S., Willis, J.R., 1985. Variational principles for inhomogeneous nonlinear media. IMA J. Appl. Math. 35, 39-54.

Taylor, G.I., 1938. Plastic strain in metals. J. Inst. Metals 62, 307-324.

Willis, J.R., 1983. The overall response of composite materials. ASME J. Appl. Mech. 50, 1202-1209. 